\title{
An Extended Solution to the Equations Describing a 3-Conductor Transmission Line
}

\author{
GEORGE ANGELOV \\ Department of Microelectronics \\ Faculty of Electronics Engineering and Technologies \\ Technical University of Sofia \\ 8 Kliment Ohridski Blvd., 1756 Sofia \\ BULGARIA
}

\begin{abstract}
The full derivation of the generalized and extended solution to the equations describing threeconductor transmission line is given in this paper; the brief results are presented in a previous paper. The considerations proceed from the C. Paul formulation of lossless transmission lines terminated by linear loads. In contrast to C. Paul, the conjoint interaction between the two lines is considered here and the influence of the receptor line is not neglected, that is the weak-coupling approximation is not applied. In result, an extended and generalized mathematical model compared the original model of $\mathrm{C}$. Paul is obtained. In particular, a mixed problem for the hyperbolic system describing the three-conductor transmission line is formulated. It is shown that the formulated mixed problem is equivalent to an initial value problem for a functional system on the boundary of hyperbolic system's domain with voltages and currents as the unknown functions in this system are the lines'. The system of functional equations can be resolved by a fixed-point method that enables us to find an approximated but explicit solution. The method elaborated in this paper might be applied also for linear as well as nonlinear boundary conditions.
\end{abstract}

Key-Words: three-conductor transmission line, electromagnetic compatibility, fixed point method, linear hyperbolic system, initial-boundary problem, mixed problem for hyperbolic system

Received: November 20, 2019. Revised: April 4, 2020. Accepted: April 19, 2020, Published: May 2, 2020.

\section{Introduction}

VLSI systems and their electromagnetic compatibility (EMC) aspects have attracted a lot of research interest (cf. [1]-[8]). In this paper an EMC model of a 3-conductor transmission line is considered taking into account the results of Clayton R. Paul [9]. In contrast to the considerations in [9], a generalized approach for solving the above problem is proposed. It is also proved that the weak coupling assumptions introduced in [9] are a particular case of the more general handling.

We obtain a general solution of the system by modeling pairwise the interacting 3-conductor transmission line introduced in [9] by keeping to the methods in [11]-[13] that were also used in other solutions such as [14], [15]. Our starting circuit is given in Fig. 1 (cf. [9]). The reference conductor for the line voltages is denoted by the ground symbol. Although it could represent an infinite ground plane, a wire, an overall shield, in our setup it is a land of a printed circuit board. The rest conductors are also lands of a printed circuit board, nevertheless they could be other objects as well. We presume the line to be an uniform and lossless line (cf. [7], [8]).

The top circuit is the generator circuit. It is terminated by a resistive load $R_{L}$ and it is driven by a voltage source with open-circuit voltage $U_{S}(t)$ and source resistance $R_{S}$. The bottom circuit is the receptor circuit. It is terminated by a resistive load $R_{N E}$ at the near end and by a resistive load $R_{F E}$ at the far end. At the terminals of the receptor circuit, the electric and magnetic fields originating by the voltage and current of the generator circuit, interact with the receptor circuit producing crosstalk voltages. 


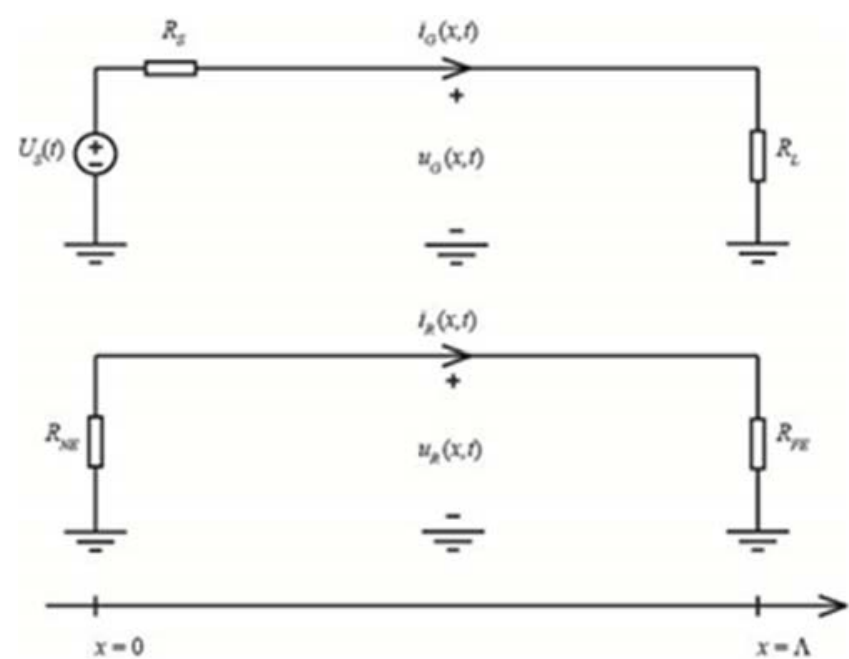

Fig. 1. Circuit of the 3-conductor transmission line.

We aim at finding a solution for the crosstalk voltages based on a system that is more than the one in [9]. That is, we proceed from the hyperbolic system (1) obtained in accordance to the TEM mode of propagation (cf. [1]-[8]). The voltages with respect to the reference conductor $u_{k}(x, t)(k=1 ; 2)$ and the currents of each circuit $i_{k}(x, t)(k=1,2)$ are functions of position $x$ and time $t$.

$$
\mid \begin{aligned}
& \frac{\partial u_{G}(x, t)}{\partial x}+L_{G} \frac{\partial i_{G}(x, t)}{\partial t}=-L_{m} \frac{\partial i_{R}(x, t)}{\partial t} \\
& \frac{\partial i_{G}(x, t)}{\partial x}+\left(C_{G}+C_{m}\right) \frac{\partial u_{G}(x, t)}{\partial t}=C_{m} \frac{\partial u_{R}(x, t)}{\partial t} \\
& \frac{\partial u_{R}(x, t)}{\partial x}+L_{R} \frac{\partial i_{R}(x, t)}{\partial t}=-L_{m} \frac{\partial i_{G}(x, t)}{\partial t} \\
& \frac{\partial i_{R}(x, t)}{\partial x}+\left(C_{R}+C_{m}\right) \frac{\partial u_{R}(x, t)}{\partial t}=C_{m} \frac{\partial u_{G}(x, t)}{\partial t}
\end{aligned}
$$

with the following boundary

$$
\begin{array}{ll}
u_{G}(0, t)=U_{S}(t)-R_{S} i_{G}(0, t), & U_{N E}=u_{R}(0, t)=-R_{N E} i_{R}(0, t) \\
u_{G}(\Lambda, t)=R_{\Lambda} i_{G}(\Lambda, t), & U_{F E}=u_{R}(\Lambda, t)=R_{F E} i_{R}(\Lambda, t)
\end{array}
$$

and initial conditions:

$$
\begin{array}{ll}
u_{G}(x, 0)=u_{G 0}(x), & u_{R}(x, 0)=u_{R 0}(x) \\
i_{G}(x, 0)=i_{G 0}(x), & i_{R}(x, 0)=i_{R 0}(x), x \in[0, \Lambda]
\end{array}
$$

Before going further, we would stress upon the fact that in our considerations, we do not apply the weak coupling assumption as opposite to [9] where this assumption is applied. This means that we do not neglect the right-hand side of (1). Therefore, our method is a more general case of (1).

Rewrite the above system (1) in the form

$$
\mid \begin{gathered}
\left(C_{G}+C_{m}\right) \frac{\partial u_{G}(x, t)}{\partial t}-C_{m} \frac{\partial u_{R}(x, t)}{\partial t}+\frac{\partial i_{G}(x, t)}{\partial x}=0 \\
-C_{m} \frac{\partial u_{G}(x, t)}{\partial t}+\left(C_{R}+C_{m}\right) \frac{\partial u_{R}(x, t)}{\partial t}+\frac{\partial i_{R}(x, t)}{\partial x}=0 \\
L_{G} \frac{\partial i_{G}(x, t)}{\partial t}+L_{m} \frac{\partial i_{R}(x, t)}{\partial t}+\frac{\partial u_{G}(x, t)}{\partial x}=0 \\
L_{m} \frac{\partial i_{G}(x, t)}{\partial t}+L_{R} \frac{\partial i_{R}(x, t)}{\partial t}+\frac{\partial u_{R}(x, t)}{\partial x}=0
\end{gathered}
$$

and introduce denotations

$$
\begin{aligned}
& u_{1}(x, t)=u_{G}(x, t) ; \quad u_{2}(x, t)=u_{R}(x, t) ; \\
& i_{1}(x, t)=i_{G}(x, t) ; \quad i_{2}(x, t)=i_{R}(x, t) \\
& C_{11}=C_{G}+C_{m}, C_{12}=C_{21}=-C_{m}, C_{22}=C_{R}+C_{m} \\
& L_{11}=L_{G}, L_{12}=L_{21}=L_{m}, L_{22}=L_{R} \\
& C=\left(\begin{array}{ll}
C_{11} & C_{12} \\
C_{12} & C_{22}
\end{array}\right), L=\left(\begin{array}{ll}
L_{11} & L_{12} \\
L_{12} & L_{22}
\end{array}\right)
\end{aligned}
$$

Then we reach the following mixed problem in the new denotations

$$
\begin{gathered}
C_{11} \frac{\partial u_{1}(x, t)}{\partial t}+C_{12} \frac{\partial u_{2}(x, t)}{\partial t}+\frac{\partial i_{1}(x, t)}{\partial x}=0, \\
C_{12} \frac{\partial u_{1}(x, t)}{\partial t}+C_{22} \frac{\partial u_{2}(x, t)}{\partial t}+\frac{\partial i_{2}(x, t)}{\partial x}=0, \\
L_{11} \frac{\partial i_{1}(x, t)}{\partial t}+L_{12} \frac{\partial i_{2}(x, t)}{\partial t}+\frac{\partial u_{1}(x, t)}{\partial x}=0, \\
L_{12} \frac{\partial i_{1}(x, t)}{\partial t}+L_{22} \frac{\partial i_{2}(x, t)}{\partial t}+\frac{\partial u_{2}(x, t)}{\partial x}=0 \\
u_{1}(0, t)=U_{S}(t)-R_{S} i_{1}(0, t), \quad U_{N E}=u_{2}(0, t)=-R_{N E} i_{2}(0, t), \\
u_{1}(\Lambda, t)=R_{L} i_{1}(\Lambda, t), \\
U_{F E}=u_{2}(\Lambda, t)=R_{F E} i_{2}(\Lambda, t) \\
u_{1}(x, 0)=u_{10}(x), \quad u_{2}(x, 0)=u_{20}(x), x \in[0, \Lambda] \\
i_{1}(x, 0)=i_{10}(x), \quad i_{2}(x, 0)=i_{20}(x), x \in[0, \Lambda]
\end{gathered}
$$

\section{Hyperbolic system transformation}

In a matrix form the above system (4) is 


$$
\begin{aligned}
& {\left[\begin{array}{cccc}
C_{11} & C_{12} & 0 & 0 \\
C_{12} & C_{22} & 0 & 0 \\
0 & 0 & L_{11} & L_{12} \\
0 & 0 & L_{12} & L_{22}
\end{array}\right]\left[\begin{array}{l}
\partial u_{1} / \partial t \\
\partial u_{2} / \partial t \\
\partial i_{1} / \partial t \\
\partial i_{2} / \partial t
\end{array}\right]+} \\
& +\left[\begin{array}{llll}
0 & 0 & 1 & 0 \\
0 & 0 & 0 & 1 \\
1 & 0 & 0 & 0 \\
0 & 1 & 0 & 0
\end{array}\right]\left[\begin{array}{l}
\partial u_{1} / \partial x \\
\partial u_{2} / \partial x \\
\partial i_{1} / \partial x \\
\partial i_{2} / \partial x
\end{array}\right]=\left[\begin{array}{l}
0 \\
0 \\
0 \\
0
\end{array}\right]
\end{aligned}
$$

Since

$$
\begin{aligned}
& \Delta_{C}=C_{11} C_{22}-C_{12}^{2}=\left(C_{G}+C_{m}\right)\left(C_{R}+C_{m}\right)-C_{m}^{2}= \\
& =C_{G} C_{R}+C_{G} C_{m}+C_{R} C_{m}>0
\end{aligned}
$$

we have to assume

Assumption (L): $\Delta_{L}=L_{G} L_{R}-L_{m}^{2}=L_{11} L_{22}-L_{12}^{2} \neq 0$.

This implies

$$
|A|=\left|\begin{array}{cccc}
C_{11} & C_{12} & 0 & 0 \\
C_{12} & C_{22} & 0 & 0 \\
0 & 0 & L_{11} & L_{12} \\
0 & 0 & L_{12} & L_{22}
\end{array}\right|=\Delta_{C} \Delta_{L} \neq 0
$$

and therefore $A^{-1}$ does exist:

$$
\begin{gathered}
A^{-1}=\frac{1}{\Delta_{C} \Delta_{L}}\left[\begin{array}{cccc}
C_{22} \Delta_{L} & -C_{12} \Delta_{L} & 0 & 0 \\
-C_{12} \Delta_{L} & C_{11} \Delta_{L} & 0 & 0 \\
0 & 0 & L_{22} \Delta_{C} & -L_{12} \Delta_{C} \\
0 & 0 & -L_{12} \Delta_{C} & L_{11} \Delta_{C}
\end{array}\right]= \\
{\left[\begin{array}{cccc}
C_{22} / \Delta_{C} & -C_{12} / \Delta_{C} & 0 & 0 \\
-C_{12} / \Delta_{C} & C_{11} / \Delta_{C} & 0 & 0 \\
0 & 0 & L_{22} / \Delta_{L} & -L_{12} / \Delta_{L} \\
0 & 0 & -L_{12} / \Delta_{L} & L_{11} / \Delta_{L}
\end{array}\right]}
\end{gathered}
$$
of

Multiplying (5) from the left by $A^{-1}$ and in view

$$
B=\left[\begin{array}{cccc}
\frac{C_{22}}{\Delta_{C}} & \frac{-C_{12}}{\Delta_{C}} & 0 & 0 \\
\frac{-C_{12}}{\Delta_{C}} & \frac{C_{11}}{\Delta_{C}} & 0 & 0 \\
0 & 0 & \frac{L_{22}}{\Delta_{L}} & \frac{-L_{12}}{\Delta_{L}} \\
0 & 0 & \frac{-L_{12}}{\Delta_{L}} & \frac{L_{11}}{\Delta_{L}}
\end{array}\right] \times\left[\begin{array}{cccc}
0 & 0 & 1 & 0 \\
0 & 0 & 0 & 1 \\
1 & 0 & 0 & 0 \\
0 & 1 & 0 & 0
\end{array}\right]=
$$

$$
=\left[\begin{array}{cccc}
0 & 0 & \frac{C_{22}}{\Delta_{C}} & \frac{-C_{12}}{\Delta_{C}} \\
0 & 0 & \frac{-C_{12}}{\Delta_{C}} & \frac{C_{11}}{\Delta_{C}} \\
\frac{L_{22}}{\Delta_{L}} & \frac{-L_{12}}{\Delta_{L}} & 0 & 0 \\
\frac{-L_{12}}{\Delta_{L}} & \frac{L_{11}}{\Delta_{L}} & 0 & 0
\end{array}\right]
$$

we obtain

$\left[\begin{array}{c}\frac{\partial u_{1}}{\partial t} \\ \frac{\partial u_{2}}{\partial t} \\ \frac{\partial i_{1}}{\partial t} \\ \frac{\partial i_{2}}{\partial t}\end{array}\right]+\left[\begin{array}{cccc}0 & 0 & \frac{C_{22}}{\Delta_{C}} & \frac{-C_{12}}{\Delta_{C}} \\ 0 & 0 & \frac{-C_{12}}{\Delta_{C}} & \frac{C_{11}}{\Delta_{C}} \\ \frac{L_{22}}{\Delta_{L}} & \frac{-L_{12}}{\Delta_{L}} & 0 & 0 \\ \frac{-L_{12}}{\Delta_{L}} & \frac{L_{11}}{\Delta_{L}} & 0 & 0\end{array}\right]\left[\begin{array}{c}\frac{\partial u_{1}}{\partial x} \\ \frac{\partial u_{2}}{\partial x} \\ \frac{\partial i_{1}}{\partial x} \\ \frac{\partial i_{2}}{\partial x}\end{array}\right]=0$

Rewrite (6) in a matrix form

$$
\frac{\partial U(x, t)}{\partial t}+B \frac{\partial U(x, t)}{\partial x}=0
$$

Substitute $U(x, t)=H Z(x, t)$ in (7):

$$
H \frac{\partial Z(x, t)}{\partial t}+B H \frac{\partial Z(x, t)}{\partial x}=0
$$

and multiplying by $H^{-1}$ we obtain

$$
\frac{\partial Z(x, t)}{\partial t}+H^{-1} B H \frac{\partial Z(x, t)}{\partial x}=0
$$

We have to find $H$ such that $H^{-1} B H=B^{\text {can }}$, where

$$
B^{\text {can }}=\left[\begin{array}{cccc}
\lambda_{1} & 0 & 0 & 0 \\
0 & \lambda_{2} & 0 & 0 \\
0 & 0 & \lambda_{3} & 0 \\
0 & 0 & 0 & \lambda_{4}
\end{array}\right]
$$

and $\lambda_{k}(k=1,2,3,4)$ are the eigen values of $B$, i.e. the roots of the equation

$$
|B-\lambda I|=\left|\begin{array}{cccc}
-\lambda & 0 & \frac{C_{22}}{\Delta_{C}} & \frac{-C_{12}}{\Delta_{C}} \\
0 & -\lambda & \frac{-C_{12}}{\Delta_{C}} & \frac{C_{11}}{\Delta_{C}} \\
\frac{L_{22}}{\Delta_{L}} & \frac{-L_{12}}{\Delta_{L}} & -\lambda & 0 \\
\frac{-L_{12}}{\Delta_{L}} & \frac{L_{11}}{\Delta_{L}} & 0 & -\lambda
\end{array}\right|=
$$




$$
\begin{aligned}
& =\lambda^{4}-\frac{L_{11} C_{11}+2 L_{12} C_{12}+L_{22} C_{22}}{\Delta_{C} \Delta_{L}} \lambda^{2}+\frac{1}{\Delta_{C} \Delta_{L}}= \\
& =\frac{\Delta_{C} \Delta_{L} \lambda^{4}-\left(L_{11} C_{11}+2 L_{12} C_{12}+L_{22} C_{22}\right) \lambda^{2}+1}{\Delta_{C} \Delta_{L}}=0
\end{aligned}
$$

We suppose

\section{Assumption (D):}

$$
\begin{aligned}
& D=\left(L_{11} C_{11}+2 L_{12} C_{12}+L_{22} C_{22}\right)^{2}- \\
& -4\left(C_{11} C_{22}-C_{12}^{2}\right)\left(L_{11} L_{22}-L_{12}^{2}\right)>0
\end{aligned}
$$

For the characteristic roots we obtain

$$
\begin{aligned}
& \lambda_{1}=\sqrt{\frac{\left(L_{11} C_{11}+2 L_{12} C_{12}+L_{22} C_{22}\right)+\sqrt{D}}{2\left(C_{11} C_{22}-C_{12}^{2}\right)\left(L_{11} L_{22}-L_{12}^{2}\right)}}, \\
& \lambda_{2}=\sqrt{\frac{\left(L_{11} C_{11}+2 L_{12} C_{12}+L_{22} C_{22}\right)-\sqrt{D}}{2\left(C_{11} C_{22}-C_{12}^{2}\right)\left(L_{11} L_{22}-L_{12}^{2}\right)}}, \\
& \lambda_{3}=-\sqrt{\frac{\left(L_{11} C_{11}+2 L_{12} C_{12}+L_{22} C_{22}\right)+\sqrt{D}}{2\left(C_{11} C_{22}-C_{12}^{2}\right)\left(L_{11} L_{22}-L_{12}^{2}\right)}}, \\
& \lambda_{4}=-\sqrt{\frac{\left(L_{11} C_{11}+2 L_{12} C_{12}+L_{22} C_{22}\right)-\sqrt{D}}{2\left(C_{11} C_{22}-C_{12}^{2}\right)\left(L_{11} L_{22}-L_{12}^{2}\right)}} .
\end{aligned}
$$

Remark. For the sake of simplicity, we could find the eigenvectors of

$$
\begin{aligned}
& \left(B^{-1}-\mu_{k} I\right) H^{(k)}=0 ; \\
& \mu_{k}=1 / \lambda_{k}, \quad H^{(k)}=\left(\xi_{1 k}, \xi_{2 k}, \xi_{3 k}, \xi_{4 k}\right)^{T}
\end{aligned}
$$

(instead of $\left.\left(B-\lambda_{k} I\right) H^{(k)}=0\right)$ because

$$
B^{-1}=\left(\begin{array}{cccc}
0 & 0 & L_{11} & L_{12} \\
0 & 0 & L_{12} & L_{22} \\
C_{11} & C_{12} & 0 & 0 \\
C_{12} & C_{22} & 0 & 0
\end{array}\right)
$$

has a simpler form than $B$.

Now we have to solve the following systems in order to obtain 4 eigenvectors $H^{(k)}(k=1,2,3,4)$

$$
\begin{aligned}
& \left(B-\lambda_{1} I\right) H^{(1)}=0, \\
& \left(B-\lambda_{2} I\right) H^{(2)}=0, \\
& \left(B-\lambda_{3} I\right) H^{(3)}=0, \\
& \left(B-\lambda_{4} I\right) H^{(4)}=0
\end{aligned}
$$

corresponding to eigenvector

$$
H^{(k)}=\left(\xi_{1 k}, \xi_{2 k}, \xi_{3 k}, \xi_{4 k}\right)^{T} ; \quad(k=1,2,3,4) .
$$

To solve (9) we have to assume:

$$
L_{12} C_{11}+L_{22} C_{12}=L_{m}\left(C_{G}+C_{m}\right)-L_{R} C_{m} \neq 0
$$

and

$$
L_{12} C_{22}+L_{11} C_{12}=L_{m}\left(C_{R}+C_{m}\right)-L_{G} C_{m} \neq 0
$$

Therefore

$$
\begin{aligned}
& \xi_{1 k}=\frac{\lambda_{k}^{2} \Delta_{L} C_{12}+L_{12}}{\lambda_{k}\left(L_{12} C_{11}+L_{22} C_{12}\right)}, \\
& \xi_{2 k}=\frac{L_{22}-\lambda_{k}^{2} \Delta_{L} C_{11}}{\lambda_{k}\left(L_{12} C_{11}+L_{22} C_{12}\right)}, \\
& \xi_{3 k}=1, \\
& \xi_{4 k}=\frac{L_{12} C_{12}+L_{22} C_{22}-\lambda_{k}^{2} \Delta_{L} \Delta_{C}}{\left(L_{12} C_{22}+L_{22} C_{12}\right)}
\end{aligned}
$$

Introduce denotations:

$$
\begin{aligned}
& \gamma_{k}=\frac{\left(1 / \lambda_{k}^{2}\right)-\left(L_{11} C_{11}+L_{12} C_{12}\right)}{L_{12} C_{11}+L_{22} C_{12}}= \\
& =\frac{1-\lambda_{k}^{2}\left(L_{11} C_{11}+L_{12} C_{12}\right)}{\lambda_{k}^{2}\left(L_{12} C_{11}+L_{22} C_{12}\right)}
\end{aligned}
$$

Note that

$$
\begin{aligned}
& \lambda_{1}>\lambda_{2}>0 ; \lambda_{3}=-\lambda_{1} ; \lambda_{4}=-\lambda_{2} ; \\
& \sqrt{D} \Rightarrow \gamma_{2}-\gamma_{1}=\frac{\sqrt{D}}{L_{12} C_{11}+L_{22} C_{12}}
\end{aligned}
$$

Then we obtain the following eigenvectors:

$$
\begin{array}{r}
H^{(1)}=\left(p_{1}, q_{1}, 1, \gamma_{1}\right)^{T}, \quad H^{(2)}=\left(p_{2}, q_{2}, 1, \gamma_{2}\right)^{T}, \\
H^{(2)}=\left(-p_{1},-q_{1}, 1, \gamma_{1}\right)^{T}, \quad H^{(1)}=\left(-p_{2},-q_{2}, 1, \gamma_{2}\right)^{T}
\end{array}
$$

where

$$
\begin{aligned}
& p_{k}=\frac{L_{12}+\lambda_{k}^{2} \Delta_{L} C_{12}}{\lambda_{k}\left(L_{12} C_{11}+L_{22} C_{12}\right)}=\lambda_{k}\left(L_{11}+L_{12} \gamma_{k}\right) \\
& q_{k}=\frac{L_{22}-\lambda_{k}^{2} \Delta_{L} C_{11}}{\lambda_{k}\left(L_{12} C_{11}+L_{22} C_{12}\right)}=\lambda_{k}\left(L_{12}+L_{22} \gamma_{k}\right), \quad(k=1,2)
\end{aligned}
$$

Thus transformation matrix becomes 


$$
H=\left[\begin{array}{cccc}
p_{1} & p_{2} & -p_{1} & -p_{2} \\
q_{1} & q_{2} & -q_{1} & -q_{2} \\
1 & 1 & 1 & 1 \\
\gamma_{1} & \gamma_{2} & \gamma_{1} & \gamma_{2}
\end{array}\right] .
$$

Since $|H|=4 \sqrt{\Delta_{L} / \Delta_{C}}\left(\gamma_{2}-\gamma_{1}\right)^{2} \neq 0$

for the inverse matrix we obtain

$$
\begin{aligned}
& H^{-1}= \\
& =\frac{1}{2\left(\gamma_{2}-\gamma_{1}\right)}\left[\begin{array}{cccc}
q_{2} \sqrt{\Delta_{L} / \Delta_{C}} & -p_{2} \sqrt{\Delta_{L} / \Delta_{C}} & \gamma_{2} & -1 \\
-q_{1} \sqrt{\Delta_{L} / \Delta_{C}} & p_{1} \sqrt{\Delta_{L} / \Delta_{C}} & -\gamma_{1} & 1 \\
-q_{2} \sqrt{\Delta_{L} / \Delta_{C}} & p_{2} \sqrt{\Delta_{L} / \Delta_{C}} & \gamma_{2} & -1 \\
q_{1} \sqrt{\Delta_{L} / \Delta_{C}} & -p_{1} \sqrt{\Delta_{L} / \Delta_{C}} & -\gamma_{1} & 1
\end{array}\right]
\end{aligned}
$$

because

$$
\left|\begin{array}{ll}
p_{1} & p_{2} \\
q_{1} & q_{2}
\end{array}\right|=\lambda_{1} \lambda_{2}\left|\left(\begin{array}{ll}
L_{11} & L_{12} \\
L_{12} & L_{22}
\end{array}\right)\left(\begin{array}{cc}
1 & 1 \\
\gamma_{1} & \gamma_{2}
\end{array}\right)\right|=\sqrt{\Delta_{L} / \Delta_{C}}\left(\gamma_{2}-\gamma_{1}\right) \text {. }
$$

\section{Boundary conditions derivation}

\section{with respect to the new variables}

Introduce new variables $U=H Z$ and $Z=H^{-1} U$, where $U=\left(u_{1}, u_{2}, i_{1}, i_{2}\right)^{T}, Z=\left(I_{1}, I_{2}, I_{3}, I_{4}\right)^{T}$.

Then

$$
\begin{aligned}
& u_{1}(x, t)=p_{1} I_{1}(x, t)+p_{2} I_{2}(x, t)-p_{1} I_{3}(x, t)-p_{2} I_{4}(x, t) \\
& u_{2}(x, t)=q_{1} I_{1}(x, t)+q_{2} I_{2}(x, t)-q_{1} I_{3}(x, t)-q_{2} I_{4}(x, t) \\
& i_{1}(x, t)=I_{1}(x, t)+I_{2}(x, t)+I_{3}(x, t)+I_{4}(x, t) \\
& i_{2}(x, t)=\gamma_{1} I_{1}(x, t)+\gamma_{2} I_{2}(x, t)+\gamma_{1} I_{3}(x, t)+\gamma_{2} I_{4}(x, t)
\end{aligned}
$$

and

$$
\begin{aligned}
& I_{1}(x, t)=\frac{1}{2\left(\gamma_{2}-\gamma_{1}\right)}\left(\begin{array}{l}
q_{2} \sqrt{\Delta_{C} / \Delta_{L}} u_{1}(x, t)- \\
-p_{2} \sqrt{\Delta_{C} / \Delta_{L}} u_{2}(x, t)+ \\
+\gamma_{2} i_{1}(x, t)-i_{2}(x, t)
\end{array}\right) \\
& I_{2}(x, t)=\frac{1}{2\left(\gamma_{2}-\gamma_{1}\right)}\left(\begin{array}{l}
-q_{1} \sqrt{\Delta_{C} / \Delta_{L}} u_{1}(x, t)+ \\
+p_{1} \sqrt{\Delta_{C} / \Delta_{L}} u_{2}(x, t)- \\
-\gamma_{1} i_{1}(x, t)+i_{2}(x, t)
\end{array}\right)
\end{aligned}
$$

$$
\begin{aligned}
& I_{3}(x, t)=\frac{1}{2\left(\gamma_{2}-\gamma_{1}\right)}\left(\begin{array}{l}
-q_{2} \sqrt{\Delta_{C} / \Delta_{L}} u_{1}(x, t)+ \\
+p_{2} \sqrt{\Delta_{C} / \Delta_{L}} u_{2}(x, t)+ \\
+\gamma_{2} i_{1}(x, t)-i_{2}(x, t)
\end{array}\right) \\
& I_{4}(x, t)=\frac{1}{2\left(\gamma_{2}-\gamma_{1}\right)}\left(\begin{array}{l}
q_{1} \sqrt{\Delta_{C} / \Delta_{L}} u_{1}(x, t)- \\
-p_{1} \sqrt{\Delta_{C} / \Delta_{L}} u_{2}(x, t)- \\
-\gamma_{1} i_{1}(x, t)+i_{2}(x, t)
\end{array}\right)
\end{aligned}
$$

Then the mixed problem (1)-(3) becomes as follows: to find a solution of the system

$$
\begin{aligned}
& \frac{\partial I_{1}(x, t)}{\partial t}+\lambda_{1} \frac{\partial I_{1}(x, t)}{\partial x}=0 \\
& \frac{\partial I_{2}(x, t)}{\partial t}+\lambda_{2} \frac{\partial I_{2}(x, t)}{\partial x}=0 \\
& \frac{\partial I_{3}(x, t)}{\partial t}-\lambda_{1} \frac{\partial I_{3}(x, t)}{\partial x}=0 \\
& \frac{\partial I_{4}(x, t)}{\partial t}-\lambda_{2} \frac{\partial I_{4}(x, t)}{\partial x}=0
\end{aligned}
$$

with initial conditions and boundary conditions in the new variables:

$$
\begin{aligned}
& I_{1}(x, 0)=\frac{1}{2\left(\gamma_{2}-\gamma_{1}\right)}\left(\begin{array}{l}
q_{2} \sqrt{\Delta_{C} / \Delta_{L}} u_{10}(x)- \\
-p_{2} \sqrt{\Delta_{C} / \Delta_{L}} u_{20}(x)+ \\
+\gamma_{2} i_{10}(x)-i_{20}(x)
\end{array}\right) \equiv I_{10}(x) \\
& I_{2}(x, 0)=\frac{1}{2\left(\gamma_{2}-\gamma_{1}\right)}\left(\begin{array}{l}
-q_{1} \sqrt{\Delta_{C} / \Delta_{L}} u_{10}(x)+ \\
+p_{1} \sqrt{\Delta_{C} / \Delta_{L}} u_{20}(x)- \\
-\gamma_{1} i_{10}(x)+i_{20}(x)
\end{array}\right) \equiv I_{20}(x) \\
& I_{3}(x, 0)=\frac{1}{2\left(\gamma_{2}-\gamma_{1}\right)}\left(\begin{array}{l}
q_{2} \sqrt{\Delta_{C} / \Delta_{L}} u_{10}(x)+ \\
+p_{2} \sqrt{\Delta_{C} / \Delta_{L}} u_{20}(x)+ \\
+\gamma_{2} i_{10}(x)-i_{20}(x) \\
I_{4} \sqrt{\Delta_{C} / \Delta_{L}} u_{10}(x)- \\
-p_{1} \sqrt{\Delta_{C} / \Delta_{L}} u_{20}(x)- \\
-\gamma_{1} i_{10}(x)+i_{20}(x)
\end{array}\right) \equiv I_{30}(x) \\
& I_{40}(x)=\frac{1}{2\left(\gamma_{2}-\gamma_{1}\right)}
\end{aligned}
$$

To obtain the boundary conditions

$u_{1}(0, t)=U_{S}(t)-R_{S} i_{1}(0, t), \quad u_{1}(\Lambda, t)=R_{\Lambda} i_{1}(\Lambda, t)$,

$U_{N E}=u_{2}(0, t)=-R_{N E} i_{2}(0, t), \quad U_{F E}=u_{2}(\Lambda, t)=R_{F E} i_{2}(\Lambda, t)$

with respect to the new variables we take into account 


$$
\begin{aligned}
& u_{1}(0, t)=p_{1} I_{1}(0, t)+p_{2} I_{2}(0, t)-p_{1} I_{3}(0, t)-p_{2} I_{4}(0, t) \\
& u_{2}(0, t)=q_{1} I_{1}(0, t)+q_{2} I_{2}(0, t)-q_{1} I_{3}(0, t)-q_{2} I_{4}(0, t) \\
& i_{1}(0, t)=I_{1}(0, t)+I_{2}(0, t)+I_{3}(0, t)+I_{4}(0, t) \\
& i_{2}(0, t)=\gamma_{1} I_{1}(0, t)+\gamma_{2} I_{2}(0, t)+\gamma_{1} I_{3}(0, t)+\gamma_{2} I_{4}(0, t)
\end{aligned}
$$

and

$$
\begin{aligned}
& u_{1}(\Lambda, t)=p_{1} I_{1}(\Lambda, t)+p_{2} I_{2}(\Lambda, t)-p_{1} I_{3}(\Lambda, t)-p_{2} I_{4}(\Lambda, t) \\
& u_{2}(\Lambda, t)=q_{1} I_{1}(\Lambda, t)+q_{2} I_{2}(\Lambda, t)-q_{1} I_{3}(\Lambda, t)-q_{2} I_{4}(\Lambda, t) \\
& i_{1}(\Lambda, t)=I_{1}(\Lambda, t)+I_{2}(\Lambda, t)+I_{3}(\Lambda, t)+I_{4}(\Lambda, t) \\
& i_{2}(\Lambda, t)=\gamma_{1} I_{1}(\Lambda, t)+\gamma_{2} I_{2}(\Lambda, t)+\gamma_{1} I_{3}(\Lambda, t)+\gamma_{2} I_{4}(\Lambda, t)
\end{aligned}
$$

\section{Consequently}

$$
\begin{aligned}
& p_{1} I_{1}(0, t)+p_{2} I_{2}(0, t)-p_{1} I_{3}(0, t)-p_{2} I_{4}(0, t)= \\
& \quad=U_{S}(t)-R_{S}\left[I_{1}(0, t)+I_{2}(0, t)+I_{3}(0, t)+I_{4}(0, t)\right] \\
& q_{1} I_{1}(0, t)+q_{2} I_{2}(0, t)-q_{1} I_{3}(0, t)-q_{2} I_{4}(0, t)= \\
& \quad=-R_{N E}\left[\gamma_{1} I_{1}(0, t)+\gamma_{2} I_{2}(0, t)+\gamma_{1} I_{3}(0, t)+\gamma_{2} I_{4}(0, t)\right] \\
& p_{1} I_{1}(\Lambda, t)+p_{2} I_{2}(\Lambda, t)-p_{1} I_{3}(\Lambda, t)-p_{2} I_{4}(\Lambda, t)= \\
& \quad=R_{\Lambda}\left[I_{1}(\Lambda, t)+I_{2}(\Lambda, t)+I_{3}(\Lambda, t)+I_{4}(\Lambda, t)\right] \\
& q_{1} I_{1}(\Lambda, t)+q_{2} I_{2}(\Lambda, t)-q_{1} I_{3}(\Lambda, t)-q_{2} I_{4}(\Lambda, t)= \\
& =R_{F E}\left[\gamma_{1} I_{1}(\Lambda, t)+\gamma_{2} I_{2}(\Lambda, t)+\gamma_{1} I_{3}(\Lambda, t)+\gamma_{2} I_{4}(\Lambda, t)\right]
\end{aligned}
$$

\section{Obtaining functional equations equivalent to the mixed problem}

Proceeding as in [11], we find the characteristics of the system (10) which are forming four families of curves

$$
\frac{d x}{d t}=\lambda_{1}, \quad \frac{d x}{d t}=\lambda_{2}, \quad \frac{d x}{d t}=-\lambda_{1}, \quad \frac{d x}{d t}=-\lambda_{2}
$$

Through each point $(x, t) \in \Pi=\{(x, t) \in[0, \Lambda] \times[0, T]\} \quad$ there are 4 characteristics: $C_{1}, C_{2}$ with positive slopes and $C_{3}$, $C_{4}$ with negative slopes. A characteristic $C_{k}(k=1,2)$ through a point $\left(0, \hat{t}_{k}\right)$ intersects the boundary $x=$ at some point $\left(\Lambda, \hat{t}_{k}+T_{k}\right)$ where $T_{k}$ can be found by integration of $\frac{d x}{d t}=\lambda_{k}$. Since the characteristic $C_{k}$ is $x-\lambda_{k} T=$ const, then the straight line through $\left(0, \hat{t}_{k}\right)$ is

$$
x-\lambda_{k} t=-\lambda_{k} \hat{t}_{k} \Rightarrow t=\frac{x}{\lambda_{k}}+\hat{t}_{k} .
$$

Setting $x=\Lambda$ and $t=\hat{t}_{k}+T_{k}$ we obtain

$$
\Lambda-\lambda_{k}\left(\hat{t}+T_{k}\right)=-\lambda_{k} \hat{t} \Rightarrow T_{k}=\Lambda / \lambda_{k} .
$$

Similarly, a characteristic $C_{p} \quad(p=3,4)$ is $x+\lambda_{p} T=$ const (with $\lambda_{3}=-\lambda_{1}, \lambda_{4}=-\lambda_{2}$ ) and the straight line through a point $\left(\Lambda, \hat{t}_{p}\right)$ is $x+\lambda_{p} t=\Lambda+\lambda_{p} \hat{t}_{p}$. It intersects $x=0$ at a point $\left(0, \hat{t}_{p}+T_{p}\right)$. Therefore

$$
\lambda_{p}\left(\hat{t}_{p}+T_{p}\right)=\Lambda+\lambda_{p} \hat{t}_{p} \Rightarrow T_{p}=\Lambda / \lambda_{p}(p=3,4),
$$

i.e. $T_{3}=T_{1}, T_{4}=T_{2}$.

Introduce directional derivatives

$$
D_{k}=\frac{\partial}{\partial t}+\frac{\partial}{\partial x} \frac{d}{d t}=\frac{\partial}{\partial t}-\lambda_{k} \frac{\partial}{\partial x}(k=1,2,3,4) .
$$

Then system (10) can be written in the form:

$$
\begin{aligned}
& D_{k} I_{k}=0(k=1,2) \\
& D_{k} I_{k}=0(k=3,4)
\end{aligned}
$$

Integrating each equation from (16) along the characteristic $C_{k}$ from $(0, t)$ to $\left(\Lambda, t+T_{k}\right)$ (where the integration is a line integral along $C_{k}$ ), we obtain

$$
I_{k}\left(\Lambda, t+T_{k}\right)=I_{k}(0, t)(t \geq 0) .
$$

In the same way, by integrating in (17) from $\left(0, t+T_{k}\right)$ to $(\Lambda, t)$, we get

$$
I_{k}(\Lambda, t)=I_{k}\left(0, t+T_{k}\right)(t \geq 0) .
$$

Present (12) in the form:

$$
\begin{aligned}
& \left(p_{1}+R_{S}\right) I_{1}(0, t)+\left(p_{2}+R_{S}\right) I_{2}(0, t)= \\
& =U_{S}(t)+\left(p_{2}-R_{S}\right) I_{3}(0, t)+\left(p_{1}-R_{S}\right) I_{4}(0, t) \\
& \left(q_{1}+R_{N E} \gamma_{1}\right) I_{1}(0, t)+\left(q_{2}+\gamma_{2} R_{N E}\right) I_{2}(0, t)= \\
& =\left(q_{2}-R_{N E} \gamma_{2}\right) I_{3}(0, t)+\left(q_{1}-R_{N E} \gamma_{1}\right) I_{4}(0, t) \\
& \left(R_{\Lambda}+p_{2}\right) I_{3}(\Lambda, t)+\left(p_{1}+R_{L}\right) I_{4}(\Lambda, t)= \\
& =\left(p_{1}-R_{\Lambda}\right) I_{1}(\Lambda, t)+\left(p_{2}-R_{L}\right) I_{2}(\Lambda, t) \\
& \left(q_{2}+R_{F E} \gamma_{2}\right) I_{3}(\Lambda, t)+\left(q_{1}+R_{F E} \gamma_{1}\right) I_{4}(\Lambda, t)= \\
& =\left(q_{1}-R_{F E} \gamma_{1}\right) I_{1}(\Lambda, t)+\left(q_{2}-R_{F E} \gamma_{2}\right) I_{2}(\Lambda, t)
\end{aligned}
$$

Since

$$
\begin{aligned}
\Delta_{12}= & \left|\begin{array}{cc}
p_{1}+R_{S} & p_{2}+R_{S} \\
q_{1}+R_{N E} \gamma_{1} & q_{2}+R_{N E} \gamma_{2}
\end{array}\right|= \\
= & \left(p_{1} q_{2}-p_{2} q_{1}\right)+\left(\gamma_{2}-\gamma_{1}\right) R_{S} R_{N E}+ \\
& \quad+\left(p_{1} \gamma_{2}-p_{2} \gamma_{1}\right) R_{N E}+\left(q_{2}-q_{1}\right) R_{S}=
\end{aligned}
$$




$$
\begin{aligned}
= & \left(\gamma_{2}-\gamma_{1}\right) \sqrt{\Delta_{L} / \Delta_{C}}+\left(\gamma_{2}-\gamma_{1}\right) R_{S} R_{N E}+ \\
& +\left(\lambda_{1}-\lambda_{2}\right) \frac{C_{22} \Delta_{L}+L_{11} \sqrt{\Delta_{L} \Delta_{C}}}{L_{12} C_{11}+L_{22} C_{12}} R_{N E}+\left(q_{2}-q_{1}\right) R_{S}= \\
= & \frac{\sqrt{D}\left(\sqrt{\Delta_{L} / \Delta_{C}}+R_{S} R_{N E}\right)}{L_{12} C_{11}+L_{22} C_{12}}+\frac{\left(\lambda_{1}-\lambda_{2}\right)}{\left(L_{12} C_{11}+L_{22} C_{12}\right)} \times \\
& \times\left(\begin{array}{l}
\left(C_{22} \Delta_{L}+L_{11} \sqrt{\Delta_{L} \Delta_{C}}\right) R_{N E}+ \\
+\frac{\left(L_{22}+\lambda_{1} \lambda_{2} \Delta_{L} C_{11}\right)}{\lambda_{1} \lambda_{2}} R_{S}
\end{array}\right) \neq \\
\neq & 0
\end{aligned}
$$

Hence, we can solve first two equations with respect to $I_{1}(0, t), I_{2}(0, t)$ which leads to

$$
I_{1}(0, t)=A_{10}(t)+A_{11} I_{3}(0, t)+A_{12} I_{4}(0, t),
$$

where

$$
\begin{aligned}
& A_{10}(t)=\frac{\left(q_{2}+R_{N E} \gamma_{2}\right) U_{S}(t)}{\Delta_{12}} ; \\
& A_{11}=\frac{2\left(p_{2} \gamma_{2} R_{N E}-q_{2} R_{S}\right)}{\Delta_{12}} ; \\
& A_{12}=\frac{p_{1} q_{2}-p_{2} q_{1}-\left(q_{2}+q_{1}\right) R_{S}}{\Delta_{12}}+ \\
& \quad+\frac{\left(p_{1} \gamma_{2}+p_{2} \gamma_{1}\right) R_{N E}+\left(\gamma_{1}-\gamma_{2}\right) R_{S} R_{N E}}{\Delta_{12}} \\
& I_{2}(0, t)=A_{20}(t)+A_{21} I_{3}(0, t)+A_{22} I_{4}(0, t),
\end{aligned}
$$

where

$$
\begin{aligned}
A_{20}(t) & =-\frac{\left(q_{1}+R_{N E} \gamma_{1}\right) U_{S}(t)}{\Delta_{12}} ; \\
A_{21}= & \frac{p_{1} q_{2}-p_{2} q_{1}+\left(q_{2}+q_{1}\right) R_{S}}{\Delta_{12}}- \\
& -\frac{\left(p_{1} \gamma_{2}+p_{2} \gamma_{1}\right) R_{N E}+\left(\gamma_{1}-\gamma_{2}\right) R_{S} R_{N E}}{\Delta_{12}} ; \\
A_{22}= & \frac{-2 p_{1} \gamma_{1}+2 q_{1} R_{S}}{\Delta_{12}}
\end{aligned}
$$

Similarly

$$
\begin{aligned}
\Delta_{34} & =\left|\begin{array}{cc}
p_{2}+R_{L} & p_{1}+R_{L} \\
q_{2}+\gamma_{2} R_{F E} & q_{1}+\gamma_{1} R_{F E}
\end{array}\right|= \\
& =\left(p_{2}+R_{L}\right)\left(q_{1}+\gamma_{1} R_{F E}\right)-\left(q_{2}+\gamma_{2} R_{F E}\right)\left(p_{1}+R_{L}\right)=
\end{aligned}
$$

$$
\begin{aligned}
& =-\frac{\sqrt{D}\left(\sqrt{\Delta_{L} / \Delta_{C}}+R_{F E} R_{L}\right)}{L_{12} C_{11}+L_{22} C_{12}}-\frac{\lambda_{1}-\lambda_{2}}{L_{12} C_{11}+L_{22} C_{12}} \times \\
& \times\left(\left(C_{22} \Delta_{L}+L_{11} \sqrt{\Delta_{L} \Delta_{C}}\right) R_{F E}+\frac{\left(L_{22}+\lambda_{1} \lambda_{2} \Delta_{L} C_{11}\right)}{\lambda_{1} \lambda_{2}} R_{L}\right) \neq \\
& \neq 0
\end{aligned}
$$

Consequently

$$
I_{3}(\Lambda, t)=B_{11} I_{1}(\Lambda, t)+B_{12} I_{2}(\Lambda, t),
$$

where

$$
\begin{aligned}
B_{11}= & \frac{2 p_{1} \gamma_{1} R_{F E}-2 q_{1} R_{L}}{\Delta_{34}} ; \\
B_{12}= & \frac{\left(p_{2} q_{1}-p_{1} q_{2}\right)-\left(q_{1}+q_{2}\right) R_{L}}{\Delta_{34}}+ \\
& +\frac{\left(p_{2} \gamma_{1}+p_{1} \gamma_{2}\right) R_{F E}+\left(\gamma_{2}-\gamma_{1}\right) R_{L} R_{F E}}{\Delta_{34}} \\
& I_{4}(\Lambda, t)=B_{21} I_{1}(\Lambda, t)+B_{22} I_{2}(\Lambda, t),
\end{aligned}
$$

where

$$
\begin{aligned}
B_{21} & =\frac{\left(p_{2} q_{1}-p_{1} q_{2}\right)+\left(q_{1}+q_{2}\right) R_{L}}{\Delta_{34}}+ \\
& +\frac{\left(\gamma_{2}-\gamma_{1}\right) R_{L} R_{F E}-\left(p_{2} \gamma_{1}+p_{1} \gamma_{2}\right) R_{F E}}{\Delta_{34}} . \\
B_{22} & =\frac{2 q_{2} R_{L}-2 p_{2} \gamma_{2} R_{F E}}{\Delta_{34}}
\end{aligned}
$$

So, we have obtained a system of functional equations

$$
\begin{aligned}
& I_{1}(0, t)=A_{10}(t)+A_{11} I_{3}(0, t)+A_{12} I_{4}(0, t) \\
& I_{2}(0, t)=A_{20}(t)+A_{21} I_{3}(0, t)+A_{22} I_{4}(0, t) \\
& I_{3}(\Lambda, t)=B_{11} I_{1}(\Lambda, t)+B_{12} I_{2}(\Lambda, t) \\
& I_{4}(\Lambda, t)=B_{21} I_{1}(\Lambda, t)+B_{22} I_{2}(\Lambda, t)
\end{aligned} .
$$

Taking into account $I_{k}\left(\Lambda, t-T_{k}\right)=I_{k}(0, t)$, $(k=3,4)$ we can rewrite the first two equations in the following way:

$$
\begin{aligned}
& I_{1}(0, t)=A_{10}(t)+A_{11} I_{3}\left(\Lambda, t-T_{3}\right)+A_{12} I_{4}\left(\Lambda, t-T_{4}\right) \\
& I_{2}(0, t)=A_{20}(t)+A_{21} I_{3}\left(\Lambda, t-T_{3}\right)+A_{22} I_{4}\left(\Lambda, t-T_{4}\right)
\end{aligned}
$$

Similarly in view of $I_{k}(\Lambda, t)=I_{k}\left(0, t-T_{k}\right)(k=1,2)$ we obtain

$$
\begin{aligned}
& I_{3}(\Lambda, t)=B_{11} I_{1}\left(0, t-T_{1}\right)+B_{12} I_{2}\left(0, t-T_{2}\right) \\
& I_{4}(\Lambda, t)=B_{21} I_{1}\left(0, t-T_{1}\right)+B_{22} I_{2}\left(0, t-T_{2}\right) .
\end{aligned}
$$


Denoting the unknown functions by

$$
\begin{array}{ll}
I_{1}(0, t) \equiv I_{1}(t), & I_{2}(0, t) \equiv I_{2}(t), \\
I_{3}(t) \equiv I_{3}(\Lambda, t), & I_{4}(t) \equiv I_{4}(\Lambda, t)
\end{array}
$$

and taking into account $T_{1}=T_{3}, T_{2}=T_{4}$ we obtain the following system:

$$
\begin{aligned}
& I_{1}(t)=A_{10}(t)+A_{11} I_{3}\left(t-T_{1}\right)+A_{12} I_{4}\left(t-T_{2}\right) \\
& I_{2}(t)=A_{20}(t)+A_{21} I_{3}\left(t-T_{1}\right)+A_{22} I_{4}\left(t-T_{2}\right) \\
& I_{3}(t)=B_{11} I_{1}\left(t-T_{1}\right)+B_{12} I_{2}\left(t-T_{2}\right) \\
& I_{4}(t)=B_{21} I_{1}\left(t-T_{1}\right)+B_{22} I_{2}\left(t-T_{2}\right)
\end{aligned}
$$

To obtain initial conditions on the intervals $\left[-T_{1}, 0\right],\left[-T_{2}, 0\right]$ one can shifted the initial functions

$$
u_{10}(x), \quad u_{20}(x), \quad i_{10}(x), \quad i_{20}(x)
$$

from the interval $[0, \Lambda]$ along the characteristics to the intervals $\left[-T_{1}, 0\right],\left[-T_{2}, 0\right]$ (cf. [12]).

The obtained functions after the above transformation on the boundary we denote by

$$
I_{10}(t), \quad I_{20}(t), \quad I_{30}(t), \quad I_{40}(t) .
$$

If $u_{10}(x), u_{20}(x), i_{10}(x), i_{20}(x)$ are periodic functions then $I_{10}(t), I_{20}(t), I_{30}(t), I_{40}(t)$ are periodic functions too.

\section{Operator presentation of the periodic problem}

Introduce the sets

$$
\begin{aligned}
& M_{1}=\left\{\begin{array}{c}
I_{1}(t) \in C_{T_{0}}[0, \infty):\left|I_{1}(t)\right| \leq I_{01} e^{\mu\left(t-k T_{0}\right)}, \\
t \in\left[k T_{0},(k+1) T_{0}\right] ; \quad I_{1}(t)=I_{10}(t), t \in\left[-T_{1}, 0\right]
\end{array}\right\}, \\
& M_{2}=\left\{\begin{array}{cc}
I_{2}(t) \in C_{T_{0}}[0, \infty):\left|I_{2}(t)\right| \leq I_{02} e^{\mu\left(t-k T_{0}\right)}, \\
t \in\left[k T_{0},(k+1) T_{0}\right] ; & I_{2}(t)=I_{20}(t), t \in\left[-T_{2}, 0\right]
\end{array}\right\}, \\
& M_{3}=\left\{\begin{array}{cc}
I_{3}(t) \in C_{T_{0}}[0, \infty):\left|I_{3}(t)\right| \leq I_{03} e^{\mu\left(t-k T_{0}\right)}, \\
t \in\left[k T_{0},(k+1) T_{0}\right] ; & I_{3}(t)=I_{30}(t), t \in\left[-T_{2}, 0\right]
\end{array}\right\}, \\
& M_{4}=\left\{\begin{array}{cc}
I_{4}(t) \in C_{T_{0}}[0, \infty): & \left|I_{4}(t)\right| \leq I_{04} e^{\mu\left(t-k T_{0}\right)}, \\
t \in\left[k T_{0},(k+1) T_{0}\right] ; & I_{4}(t)=I_{40}(t), t \in\left[-T_{1}, 0\right]
\end{array}\right\},
\end{aligned}
$$

$(k=0,1,2, \ldots)(k=0,1,2, \ldots)$, where $C_{T_{0}}[0, \infty)$ is the set of all continuous $T_{0}$-periodic functions and $I_{0 k}, T_{0}, \mu$ are positive constants and $\mu T_{0}=\mu_{0}=$ const.

We use the technique of fixed point theory in uniform spaces (cf. [13]). For that purpose, we introduce a saturated family of pseudo-metrics in the Cartesian product

$$
\begin{gathered}
M=M_{1} \times M_{2} \times M_{3} \times M_{4}: \\
\rho^{(k)}\left(I_{n}, \bar{I}_{n}\right)= \\
=\max \left\{\left|I_{n}(t)-\bar{I}_{n}(t)\right| e^{-\mu\left(t-k T_{0}\right)}: t \in\left[k T_{0},(k+1) T_{0}\right]\right\} \\
(n=1,2,3,4 ; k=0,1,2, \ldots),
\end{gathered}
$$

where the index set of this family consists of all ordered fours

$$
\left(p_{1}, p_{2}, p_{3}, p_{4}\right) \in N_{0} \times N_{0} \times N_{0} \times N_{0} ; N_{0}=\{0,1,2, \ldots\}
$$

corresponding to the initial points of the intervals

$$
\begin{aligned}
& {\left[p_{1} T_{0},\left(p_{1}+1\right) T_{0}\right] \times\left[p_{2} T_{0},\left(p_{2}+1\right) T_{0}\right] \times} \\
& \times\left[p_{3} T_{0},\left(p_{3}+1\right) T_{0}\right] \times\left[p_{4} T_{0},\left(p_{4}+1\right) T_{0}\right]
\end{aligned} .
$$

Introduce maps $j_{n}(k): N_{0} \rightarrow N_{0} \quad(n=1,2)$ in the following way:

$$
j_{1}: N_{0} \rightarrow N_{0} ;\left[k T_{0},(k+1) T_{0}\right] \rightarrow\left[k T_{0}-T_{1},(k+1) T_{0}-T_{1}\right]
$$

We suppose that $T_{p}=m_{p} T_{0},(p=1,2)$. Therefore

$$
\left[k T_{0}-T_{p},(k+1) T_{0}-T_{p}\right]=\left[\left(k-m_{p}\right) T_{0},\left(k+1-m_{p}\right) T_{0}\right]
$$

and then $j_{p}(k): k \rightarrow k-m_{p}$ provided $k-m_{p} \geq 0$; $j_{p}{ }^{m}(k)=j_{p}\left(j_{p}^{m-1}(k)\right), j_{p}{ }^{0}(k)=k$. The definition of $j_{p}$ implies that $j_{p}^{m}(k) \in N_{0}$ only for finite $m$.

Define

$$
\begin{aligned}
& j\left(p_{1}, p_{2}, p_{3}, p_{4}\right)=\left(j_{1}\left(p_{1}\right), j_{2}\left(p_{2}\right), j_{3}\left(p_{3}\right), j_{4}\left(p_{4}\right)\right) \\
& j^{2}\left(p_{1}, p_{2}, p_{3}, p_{4}\right)=\left(j_{1}^{2}\left(p_{1}\right), j_{2}^{2}\left(p_{2}\right), j_{3}^{2}\left(p_{3}\right), j_{4}^{2}\left(p_{4}\right)\right)
\end{aligned}
$$

In particular,

$$
j(p, p, p, p)=\left(j_{1}(p), j_{2}(p), j_{3}(p), j_{4}(p)\right)
$$

The set $M$ turns out into a complete uniform space with a saturated family of pseudometrics (cf. [13]) 


$$
\begin{aligned}
& \rho_{\left(p_{1}, p_{2}, p_{1}, p_{2}\right)}\left(\left(I_{1}, I_{2}, I_{3}, I_{4}\right),\left(\bar{I}_{1}, \bar{I}_{2}, \bar{I}_{3}, \bar{I}_{4}\right)\right)= \\
& =\rho^{\left(p_{1}\right)}\left(I_{1}, \bar{I}_{1}\right)+\rho^{\left(p_{2}\right)}\left(I_{2}, \bar{I}_{2}\right)+\rho^{\left(p_{1}\right)}\left(I_{3}, \bar{I}_{3}\right)+\rho^{\left(p_{2}\right)}\left(I_{4}, \bar{I}_{4}\right)
\end{aligned}
$$

Now we formulate the main problem: to find a $T_{0}$-periodic solution of:

$$
\begin{aligned}
& I_{1}(t)=A_{10}(t)+A_{11} I_{3}\left(t-T_{1}\right)+A_{12} I_{4}\left(t-T_{2}\right) \\
& I_{2}(t)=A_{20}(t)+A_{21} I_{3}\left(t-T_{1}\right)+A_{22} I_{4}\left(t-T_{2}\right) \\
& I_{3}(t)=B_{11} I_{1}\left(t-T_{1}\right)+B_{12} I_{2}\left(t-T_{2}\right) \\
& I_{4}(t)=B_{21} I_{1}\left(t-T_{1}\right)+B_{22} I_{2}\left(t-T_{2}\right)
\end{aligned}
$$

where

$$
\begin{array}{ll}
I_{1}(t)=I_{10}(t), t \in\left[-T_{1}, 0\right], & I_{2}(t)=I_{20}(t), t \in\left[-T_{2}, 0\right], \\
I_{3}(t)=I_{30}(t), t \in\left[-T_{1}, 0\right], & I_{4}(t)=I_{40}(t), t \in\left[-T_{2}, 0\right]
\end{array}
$$

We define an operator with components $B=\left(B_{1}\right.$, $\left.B_{2}, B_{3}, B_{4}\right)$ by the formulas:

$$
\begin{aligned}
& B_{1}\left(I_{1}, I_{2}, I_{3}, I_{4}\right)(t):=\left\{\begin{array}{r}
A_{10}(t)+A_{11} I_{3}\left(t-T_{1}\right)+A_{12} I_{4}\left(t-T_{2}\right) \\
I_{3}(t)=I_{30}(t), t \in\left[-T_{1}, 0\right] \\
I_{4}(t)=I_{40}(t), t \in\left[-T_{2}, 0\right]
\end{array}\right. \\
& t \in[0, \infty) \\
& B_{2}\left(I_{1}, I_{2}, I_{3}, I_{4}\right)(t):=\left\{\begin{array}{r}
A_{20}(t)+A_{21} I_{3}\left(t-T_{1}\right)+A_{22} I_{4}\left(t-T_{2}\right) \\
I_{3}(t)=I_{30}(t), t \in\left[-T_{1}, 0\right] \\
I_{4}(t)=I_{40}(t), t \in\left[-T_{2}, 0\right]
\end{array}\right. \\
& t \in[0, \infty) \\
& B_{3}\left(I_{1}, I_{2}, I_{3}, I_{4}\right)(t):=\left\{\begin{array}{r}
B_{11} I_{1}\left(t-T_{1}\right)+B_{12} I_{2}\left(t-T_{2}\right) \\
I_{1}(t)=I_{10}(t), t \in\left[-T_{1}, 0\right] \\
I_{2}(t)=V_{20}(t), t \in\left[-T_{2}, 0\right]
\end{array}\right. \\
& t \in[0, \infty) \\
& B_{4}\left(I_{1}, I_{2}, I_{3}, I_{4}\right)(t):=\left\{\begin{array}{r}
B_{21} I_{1}\left(t-T_{1}\right)+B_{22} I_{2}\left(t-T_{2}\right) \\
I_{1}(t)=I_{10}(t), t \in\left[-T_{1}, 0\right] \\
I_{2}(t)=I_{20}(t), t \in\left[-T_{2}, 0\right]
\end{array} .\right. \\
& t \in[0, \infty)
\end{aligned}
$$

\section{Existence-uniqueness of periodic solution}

The main result is:

Theorem 1. Let the following conditions be fulfilled:

$$
\begin{aligned}
& I_{10}(.), I_{30}(.) \in C_{T_{0}}^{1}\left[-T_{1}, 0\right], \quad I_{20}(.), I_{40}(.) \in C_{T_{0}}^{1}\left[-T_{2}, 0\right] \\
& U_{S}(.) \in C_{T_{0}}[0, \infty), \quad \breve{U}_{S}=\max \left\{\left|U_{S}(t)\right|: t \in\left[0, T_{0}\right]\right\}
\end{aligned}
$$

Assumptions (D) and (L) are valid;

$T_{1}=m_{1} T_{0}, T_{2}=m_{2} T_{0}$ for positive integers $m_{1}, m_{2}$

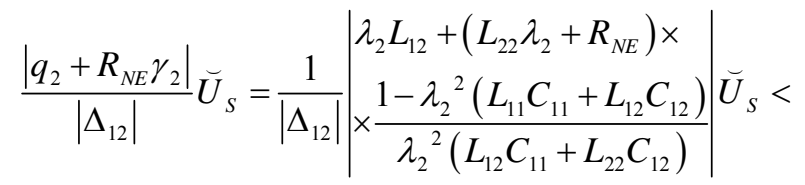

$$
<\min \left\{I_{10}, I_{20}\right\}
$$

$$
\begin{aligned}
\frac{\left|q_{1}+R_{N E} \gamma_{1}\right|}{\left|\Delta_{12}\right|} \breve{U}_{S} & =\frac{1}{\left|\Delta_{12}\right|}\left|\begin{array}{c}
\lambda_{1} L_{12}+\left(L_{22} \lambda_{1}+R_{N E}\right) \times \\
1-\lambda_{1}^{2}\left(L_{11} C_{11}+L_{12} C_{12}\right) \\
\lambda_{1}^{2}\left(L_{12} C_{11}+L_{22} C_{12}\right)
\end{array}\right| \breve{U}_{S}< \\
& <\min \left\{I_{10}, I_{20}\right\}
\end{aligned}
$$
(19).

Proof: The set $M_{1} \times M_{2} \times M_{3} \times M_{4}$ is a uniform space with the above saturated family of pseudometrics. In view of $\lambda_{1}>\lambda_{2} \Rightarrow \Lambda / \lambda_{2}>\Lambda / \lambda_{1} \Rightarrow T_{2}>T_{1}$ we show that $B$ maps $M_{1} \times M_{2} \times M_{3} \times M_{4}$ into itself. It is easy to verify that all components of the operator $B$ are periodic functions.

For $t \in\left[k T_{0},(k+1) T_{0}\right]$ and sufficiently large $\mu>0$, having in mind (12), (13) we obtain:

$$
\begin{aligned}
\mid B_{1}^{(k)} & \left(I_{1}, I_{2}, I_{3}, I_{4}\right)(t) \mid \leq \\
& \leq\left|A_{10}(t)\right|+\left|A_{11}\right|\left|I_{3}\left(t-T_{1}\right)\right|+\left|A_{12}\right|\left|I_{4}\left(t-T_{2}\right)\right| \leq \\
& \leq\left|A_{10}(t)\right|+\left|A_{11}\right| I_{30} e^{\mu\left(t-T_{1}-k T_{0}\right)}+\left|A_{12}\right| I_{40} e^{\mu\left(t-T_{2}-k T_{0}\right)} \leq \\
& \leq e^{\mu\left(t-k T_{0}\right)}\left(\frac{\left|q_{2}+R_{N E} \gamma_{2}\right|}{\left|\Delta_{12}\right|} \breve{U}_{S}+\left|A_{11}\right| I_{30} e^{-\mu T_{1}}+\left|A_{12}\right| I_{40} e^{-\mu T_{2}}\right) \leq \\
& \leq e^{\mu\left(t-k T_{0}\right)} I_{10}
\end{aligned}
$$




$$
\begin{aligned}
\mid B_{2}^{(k)} & \left(I_{1}, I_{2}, I_{3}, I_{4}\right)(t) \mid \leq \\
& \leq\left|A_{20}(t)\right|+\left|A_{21}\right|\left|I_{3}\left(t-T_{1}\right)\right|+\left|A_{22}\right|\left|I_{4}\left(t-T_{2}\right)\right| \leq \\
& \leq \frac{\left|q_{1}+R_{N E} \gamma_{1}\right| \breve{U}_{S}}{\left|\Delta_{12}\right|}+\left|A_{21}\right| I_{30} e^{\mu\left(t-T_{1}-k T_{0}\right)}+\left|A_{22}\right| I_{40} e^{\mu\left(t-T_{2}-k T_{0}\right)} \leq \\
& \leq e^{\mu\left(t-k T_{0}\right)}\left(\frac{\left|q_{1}+R_{N E} \gamma_{1}\right| \breve{U}_{S}}{\left|\Delta_{12}\right|}+\left|A_{21}\right| I_{30} e^{-\mu T_{1}}+\left|A_{22}\right| I_{40} e^{-\mu T_{2}}\right) \leq \\
& \leq e^{\mu\left(t-k T_{0}\right)} I_{20}
\end{aligned}
$$

$$
\begin{aligned}
\mid B_{3}^{(k)} & \left(I_{1}, I_{2}, I_{3}, I_{4}\right)(t) \mid \leq \\
& \leq\left|B_{11}\right|\left|I_{1}\left(t-T_{1}\right)\right|+\left|B_{12}\right|\left|I_{2}\left(t-T_{2}\right)\right| \leq \\
& \leq\left|B_{11}\right| I_{10} e^{\mu\left(t-T_{1}-k T_{0}\right)}+\left|B_{12}\right| I_{20} e^{\mu\left(t-T_{2}-k T_{0}\right)} \leq \\
& \leq e^{\mu\left(t-k T_{0}\right)}\left(\left|B_{11}\right| I_{10} e^{-\mu T_{1}}+\left|B_{12}\right| I_{20} e^{-\mu T_{2}}\right) \leq \\
& \leq e^{\mu\left(t-k T_{0}\right)} I_{30}
\end{aligned}
$$

$$
\begin{aligned}
\mid B_{4}^{(k)} & \left(I_{1}, I_{2}, I_{3}, I_{4}\right)(t) \mid \leq \\
& \leq\left|B_{21}\right|\left|I_{1}\left(t-T_{1}\right)\right|+\left|B_{22}\right|\left|I_{2}\left(t-T_{2}\right)\right| \leq \\
& \leq\left|B_{21}\right| I_{10} e^{\mu\left(t-T_{1}-k T_{0}\right)}+\left|B_{22}\right| I_{20} e^{\mu\left(t-T_{2}-k T_{0}\right)} \leq \\
& \leq e^{\mu\left(t-k T_{0}\right)}\left(\left|B_{21}\right| I_{10} e^{-\mu T_{1}}+\left|B_{22}\right| I_{20} e^{-\mu T_{2}}\right) \leq \\
& \leq e^{\mu\left(t-k T_{0}\right)} I_{40}
\end{aligned}
$$

It remains to show that $B$ is contractive operator. We notice that $T_{2}>T_{1} \Rightarrow-\mu T_{2}<-\mu T_{1} \Rightarrow e^{-\mu T_{2}}<e^{-\mu T_{1}}$

Then

$$
\begin{aligned}
& \max \left\{\begin{array}{l}
\left|A_{11}\right| e^{-\mu T_{1}}+\left|A_{12}\right| e^{-\mu T_{2}} ;\left|A_{21}\right| e^{-\mu T_{1}}+\left|A_{22}\right| e^{-\mu T_{2}} ; \\
\left|B_{11}\right| e^{-\mu T_{1}}+\left|B_{12}\right| e^{-\mu T_{2}} ;\left|B_{21}\right| e^{-\mu T_{1}}+\left|B_{22}\right| e^{-\mu T_{2}}
\end{array}\right\} \leq \\
& \leq e^{-\mu T_{1}} \max \left\{\begin{array}{l}
\left|A_{11}\right|+\left|A_{12}\right| ;\left|A_{21}\right|+\left|A_{22}\right| ; \\
\left|B_{11}\right|+\left|B_{12}\right| ;\left|B_{21}\right|+\left|B_{22}\right|
\end{array}\right\}=K(\mu)
\end{aligned}
$$

and for $t \in\left[k T_{0},(k+1) T_{0}\right]$ we have

$$
\begin{aligned}
& \left|B_{1}^{(k)}\left(I_{3}, I_{4}\right)(t)-B_{1}^{(k)}\left(\bar{I}_{3}, \bar{I}_{4}\right)(t)\right| \leq \\
& \leq\left|A_{11}\right|\left|I_{3}\left(t-T_{1}\right)-\bar{I}_{3}\left(t-T_{1}\right)\right|+\left|A_{12}\right|\left|I_{4}\left(t-T_{2}\right)-\bar{I}_{4}\left(t-T_{2}\right)\right| \leq \\
& \leq\left|A_{11}\right|\left|I_{3}\left(t-T_{1}\right)-\bar{I}_{3}\left(t-T_{1}\right)\right| e^{-\mu\left(t-k T_{0}-T_{1}\right)} e^{\mu\left(t-k T_{0}-T_{1}\right)}+ \\
& \quad+\left|A_{12}\right|\left|I_{4}\left(t-T_{2}\right)-\bar{I}_{4}\left(t-T_{2}\right)\right| e^{-\mu\left(t-k T_{0}-T_{2}\right)} e^{\mu\left(t-k T_{0}-T_{2}\right)} \leq \\
& \leq e^{\mu\left(t-k T_{0}\right)}\left[\left|A_{11}\right| e^{-\mu T_{1}} \rho^{j_{1}(k)}\left(I_{3}, \bar{I}_{3}\right)+\left|A_{12}\right| e^{-\mu T_{2}} \rho^{j_{2}(k)}\left(I_{4}, \bar{I}_{4}\right)\right]
\end{aligned}
$$

$$
\begin{aligned}
& \rho^{(k)}\left(B_{1}^{(k)}\left(I_{3}, I_{4}\right), B_{1}^{(k)}\left(\bar{I}_{3}, \bar{I}_{4}\right)\right) \leq \\
& \leq\left|A_{11}\right| e^{-\mu T_{1}} \rho^{j_{1}(k)}\left(I_{3}, \bar{I}_{3}\right)+\left|A_{12}\right| e^{-\mu T_{2}} \rho^{j_{2}(k)}\left(I_{4}, \bar{I}_{4}\right) \leq \\
& \leq\left(\left|A_{11}\right| e^{-\mu T_{1}}+\left|A_{12}\right| e^{-\mu T_{2}}\right)\left(\rho^{j_{1}(k)}\left(I_{3}, \bar{I}_{3}\right)+\rho^{j_{2}(k)}\left(I_{4}, \bar{I}_{4}\right)\right) \leq \\
& \leq\left(\left|A_{11}\right| e^{-\mu T_{1}}+\left|A_{12}\right| e^{-\mu T_{2}}\right)\left(\begin{array}{l}
\rho^{j_{1}(k)}\left(I_{1}, \bar{I}_{1}\right)+\rho^{j_{2}(k)}\left(I_{2}, \bar{I}_{2}\right)+ \\
+\rho^{j_{1}(k)}\left(I_{3}, \bar{I}_{3}\right)+\rho^{j_{2}(k)}\left(I_{4}, \bar{I}_{4}\right)
\end{array}\right) \leq \\
& \leq K(\mu)\left(\begin{array}{l}
\rho^{j_{1}(k)}\left(I_{1}, \bar{I}_{1}\right)+\rho^{j_{2}(k)}\left(I_{2}, \bar{I}_{2}\right)+ \\
+\rho^{j_{1}(k)}\left(I_{3}, \bar{I}_{3}\right)+\rho^{j_{2}(k)}\left(I_{4}, \bar{I}_{4}\right)
\end{array}\right) \\
&\left|B_{2}^{(k)}\left(I_{3}, I_{4}\right)(t)-B_{2}^{(k)}\left(\bar{I}_{3}, \bar{I}_{4}\right)(t)\right| \leq \\
& \leq\left|A_{21}\right|\left|I_{3}\left(t-T_{1}\right)-\bar{I}_{3}\left(t-T_{1}\right)\right|+\left|A_{22}\right|\left|I_{4}\left(t-T_{2}\right)-\bar{I}_{4}\left(t-T_{2}\right)\right| \leq \\
& \leq\left|A_{21}\right|\left|I_{3}\left(t-T_{1}\right)-\bar{I}_{3}\left(t-T_{1}\right)\right| e^{-\mu\left(t-k T_{0}-T_{1}\right)} e^{\mu\left(t-k T_{0}-T_{1}\right)}+ \\
& \quad+\left|A_{22}\right|\left|I_{4}\left(t-T_{2}\right)-\bar{I}_{4}\left(t-T_{2}\right)\right| e^{-\mu\left(t-k T_{0}-T_{2}\right)} e^{\mu\left(t-k T_{0}-T_{2}\right)} \\
& \leq e^{\mu\left(t-k T_{0}\right)}\left[\left|A_{21}\right| e^{-\mu T_{1}} \rho^{j_{1}(k)}\left(I_{3}, \bar{I}_{3}\right)+\left|A_{22}\right| e^{-\mu T_{2}} \rho^{j_{2}(k)}\left(I_{4}, \bar{I}_{4}\right)\right]
\end{aligned}
$$

which implies

$$
\begin{aligned}
\rho^{(k)} & \left(B_{2}^{(k)}\left(I_{3}, I_{4}\right), B_{2}^{(k)}\left(\bar{I}_{3}, \bar{I}_{4}\right)\right) \leq \\
& \leq\left|A_{21}\right| e^{-\mu T_{1}} \rho^{j_{1}(k)}\left(I_{3}, \bar{I}_{3}\right)+\left|A_{22}\right| e^{-\mu T_{2}} \rho^{j_{2}(k)}\left(I_{4}, \bar{I}_{4}\right) \leq \\
& \leq\left(\left|A_{21}\right| e^{-\mu T_{1}}+\left|A_{22}\right| e^{-\mu T_{2}}\right)\left(\rho^{j_{1}(k)}\left(I_{3}, \bar{I}_{3}\right)+\rho^{j_{2}(k)}\left(I_{4}, \bar{I}_{4}\right)\right) \leq \\
& \leq\left(\left|A_{21}\right| e^{-\mu T_{1}}+\left|A_{22}\right| e^{-\mu T_{2}}\right)\left(\begin{array}{l}
\rho^{j_{1}(k)}\left(I_{1}, \bar{I}_{1}\right)+\rho^{j_{2}(k)}\left(I_{2}, \bar{I}_{2}\right)+ \\
+\rho^{j_{1}(k)}\left(I_{3}, \bar{I}_{3}\right)+\rho^{j_{2}(k)}\left(I_{4}, \bar{I}_{4}\right)
\end{array}\right) \leq \\
& \leq K(\mu)\left(\begin{array}{l}
\rho^{j_{1}(k)}\left(I_{1}, \bar{I}_{1}\right)+\rho^{j_{2}(k)}\left(I_{2}, \bar{I}_{2}\right)+ \\
+\rho^{j_{1}(k)}\left(I_{3}, \bar{I}_{3}\right)+\rho^{j_{2}(k)}\left(I_{4}, \bar{I}_{4}\right)
\end{array}\right)
\end{aligned}
$$

Further on we have

$$
\begin{aligned}
& \left|B_{3}^{(k)}\left(I_{1}, I_{2}\right)(t)-B_{3}^{(k)}\left(\bar{I}_{1}, \bar{I}_{2}\right)(t)\right| \leq \\
& \quad \leq\left|B_{11}\right|\left|I_{1}\left(t-T_{1}\right)-\bar{I}_{1}\left(t-T_{1}\right)\right|+\left|B_{12}\right|\left|I_{2}\left(t-T_{2}\right)-\bar{I}_{2}\left(t-T_{2}\right)\right| \leq \\
& \leq\left|B_{11}\right|\left|I_{1}\left(t-T_{1}\right)-\bar{I}_{1}\left(t-T_{1}\right)\right| e^{-\mu\left(t-k T_{0}-T_{1}\right)} e^{\mu\left(t-k T_{0}-T_{1}\right)}+ \\
& \quad \quad|| B_{12}|| I_{2}\left(t-T_{2}\right)-\bar{I}_{2}\left(t-T_{2}\right) \mid e^{-\mu\left(t-k T_{0}-T_{2}\right)} e^{\mu\left(t-k T_{0}-T_{2}\right)} \leq \\
& \quad \leq e^{\mu\left(t-k T_{0}\right)}\left[\left|B_{11}\right| e^{-\mu T_{1}} \rho^{j_{1}(k)}\left(I_{1}, \bar{I}_{1}\right)+\left|B_{12}\right| e^{-\mu T_{2}} \rho^{j_{2}(k)}\left(I_{2}, \bar{I}_{2}\right)\right]
\end{aligned}
$$

which implies

that implies 


$$
\begin{aligned}
\rho^{(k)} & \left(B_{3}^{(k)}\left(I_{1}, I_{2}\right), B_{3}^{(k)}\left(\bar{I}_{1}, \bar{I}_{2}\right)\right) \leq \\
& \leq\left|B_{11}\right| e^{-\mu T_{1}} \rho^{j_{1}(k)}\left(I_{1}, \bar{I}_{1}\right)+\left|B_{12}\right| e^{-\mu T_{2}} \rho^{j_{2}(k)}\left(I_{2}, \bar{I}_{2}\right) \leq \\
& \leq\left(\left|B_{11}\right| e^{-\mu T_{1}}+\left|B_{12}\right| e^{-\mu T_{2}}\right)\left(\rho^{j_{1}(k)}\left(I_{1}, \bar{I}_{1}\right)+\rho^{j_{2}(k)}\left(I_{2}, \bar{I}_{2}\right)\right) \leq \\
& \leq\left(\left|B_{11}\right| e^{-\mu T_{1}}+\left|B_{12}\right| e^{-\mu T_{2}}\right)\left(\begin{array}{l}
\rho^{j_{1}(k)}\left(I_{1}, \bar{I}_{1}\right)+\rho^{j_{2}(k)}\left(I_{2}, \bar{I}_{2}\right)+ \\
+\rho^{j_{2}(k)}\left(I_{3}, \bar{I}_{3}\right)+\rho^{j_{1}(k)}\left(I_{4}, \bar{I}_{4}\right)
\end{array}\right) \leq \\
& \leq K(\mu)\left(\begin{array}{l}
\rho^{j_{1}(k)}\left(I_{1}, \bar{I}_{1}\right)+\rho^{j_{2}(k)}\left(I_{2}, \bar{I}_{2}\right)+ \\
+\rho^{j_{2}(k)}\left(I_{3}, \bar{I}_{3}\right)+\rho^{j_{1}(k)}\left(I_{4}, \bar{I}_{4}\right)
\end{array}\right)
\end{aligned}
$$

Finally, we have

$$
\begin{aligned}
& \left|B_{4}^{(k)}\left(I_{1}, I_{2}\right)(t)-B_{4}^{(k)}\left(\bar{I}_{3}, \bar{I}_{4}\right)(t)\right| \leq \\
& \quad \leq\left|B_{21}\right|\left|I_{1}\left(t-T_{1}\right)-\bar{I}_{11}\left(t-T_{1}\right)\right|+\left|B_{22}\right|\left|I_{22}\left(t-T_{2}\right)-\bar{I}_{2}\left(t-T_{2}\right)\right| \leq \\
& \leq\left|B_{21}\right|\left|I_{1}\left(t-T_{1}\right)-\bar{I}_{1}\left(t-T_{1}\right)\right| e^{-\mu\left(t-k T_{0}-T_{1}\right)} e^{\mu\left(t-k T_{0}-T_{1}\right)}+ \\
& \quad+\left|B_{22}\right|\left|I_{2}\left(t-T_{2}\right)-\bar{I}_{2}\left(t-T_{2}\right)\right| e^{-\mu\left(t-k T_{0}-T_{2}\right)} e^{\mu\left(t-k T_{0}-T_{2}\right)} \leq \\
& \quad \leq e^{\mu\left(t-T_{0}\right)}\left[\left|B_{21}\right| e^{-\mu T_{1}} \rho^{j_{1}(k)}\left(I_{1}, \bar{I}_{1}\right)+\left|B_{22}\right| e^{-\mu T_{2}} \rho^{j_{2}(k)}\left(I_{2}, \bar{I}_{2}\right)\right]
\end{aligned}
$$

which implies

$$
\begin{aligned}
\rho^{(k)} & \left(B_{4}^{(k)}\left(I_{1}, I_{2}\right), B_{4}^{(k)}\left(\bar{I}_{1}, \bar{I}_{2}\right)\right) \leq \\
& \leq\left|B_{21}\right| e^{-\mu T_{1}} \rho^{j_{1}(k)}\left(I_{1}, \bar{I}_{1}\right)+\left|B_{22}\right| e^{-\mu T_{2}} \rho^{j_{2}(k)}\left(I_{2}, \bar{I}_{2}\right) \leq \\
& \leq\left(\left|B_{21}\right| e^{-\mu T_{1}}+\left|B_{22}\right| e^{-\mu T_{2}}\right)\left(\rho^{j_{1}(k)}\left(I_{1}, \bar{I}_{1}\right)+\rho^{j_{2}(k)}\left(I_{2}, \bar{I}_{2}\right)\right) \leq \\
& \leq\left(\left|B_{21}\right| e^{-\mu T_{1}}+\left|B_{22}\right| e^{-\mu T_{2}}\right)\left(\begin{array}{l}
\rho^{j_{1}(k)}\left(I_{1}, \bar{I}_{1}\right)+\rho^{j_{2}(k)}\left(I_{2}, \bar{I}_{2}\right)+ \\
+\rho^{j_{1}(k)}\left(I_{3}, \bar{I}_{3}\right)+\rho^{j_{2}(k)}\left(I_{4}, \bar{I}_{4}\right)
\end{array}\right) \leq \\
& \leq K(\mu)\left(\begin{array}{l}
\rho^{j_{1}(k)}\left(I_{1}, \bar{I}_{1}\right)+\rho^{j_{2}(k)}\left(I_{2}, \bar{I}_{2}\right)+ \\
+\rho^{j_{1}(k)}\left(I_{3}, \bar{I}_{3}\right)+\rho^{j_{2}(k)}\left(I_{4}, \bar{I}_{4}\right)
\end{array}\right)
\end{aligned}
$$

Therefore

$$
\begin{gathered}
\rho^{(k)}\left(\begin{array}{l}
\left(B_{1}^{(k)}\left(I_{3}, I_{4}\right), B_{2}^{(k)}\left(I_{3}, I_{4}\right), B_{3}^{(k)}\left(I_{1}, I_{2}\right), B_{4}^{(k)}\left(I_{1}, I_{2}\right)\right), \\
\left(B_{1}^{(k)}\left(\bar{I}_{3}, \bar{I}_{4}\right), B_{2}^{(k)}\left(\bar{I}_{3}, \bar{I}_{4}\right), B_{3}^{(k)}\left(\bar{I}_{1}, \bar{I}_{2}\right), B_{4}^{(k)}\left(\bar{I}_{1}, \bar{I}_{2}\right)\right)
\end{array}\right) \leq \\
\quad \leq 4 K(\mu)\left(\begin{array}{l}
\rho^{j_{1}(k)}\left(I_{1}, \bar{I}_{1}\right)+\rho^{j_{2}(k)}\left(I_{2}, \bar{I}_{2}\right)+ \\
+\rho^{j_{1}(k)}\left(I_{3}, \bar{I}_{3}\right)+\rho^{j_{2}(k)}\left(I_{4}, \bar{I}_{4}\right)
\end{array}\right)
\end{gathered}
$$

It is easy to see that $j^{n}(k, k, k, k)<Q(k)<\infty$ $(n=1,2, \ldots)$ is uniformly bounded by $n ; Q$ is a positive constant not depending on $n$. Indeed, every interval goes to the left from the initial point after a finite number $n$ of iteration of $j$. This means that the operator $B$ is contractive one in the sense of definition from [13] and has a unique fixed point $\left(I_{1}(t), I_{2}(t), I_{3}(t), I_{4}(t)\right)$, which is a solution of (19).

Finally, we note that the solution can be approximated by a sequence of successive approximations with advanced prescribed accuracy.

Theorem 1 is thus proved.

\section{Results validation}

Since our goal is to find $U_{N E}=u_{2}(0, t) ; U_{F E}=u_{2}(\Lambda, t)$ we have (cf. (12), (13)): $u_{2}(0, t)=q_{1} I_{1}(0, t)+q_{2} I_{2}(0, t)-q_{1} I_{3}(0, t)-q_{2} I_{4}(0, t)=$ $=q_{1} I_{1}(t)+q_{2} I_{2}(t)-q_{1} I_{3}\left(t-T_{1}\right)-q_{2} I_{4}\left(t-T_{2}\right)$

$u_{2}(\Lambda, t)=q_{1} I_{1}(\Lambda, t)+q_{2} I_{2}(\Lambda, t)-q_{1} I_{3}(\Lambda, t)-q_{2} I_{4}(0, t)=$ $=q_{1} I_{1}\left(t-T_{1}\right)+q_{2} I_{2}\left(t-T_{2}\right)-q_{1} I_{3}(t)-q_{2} I_{4}(t)$

where $\left(I_{1}(t), I_{2}(t), I_{3}(t), I_{4}(t)\right)$ is the solution obtained in the above theorem.

We have to check the conditions of our Theorem 1 referring to the data from [9] :

$L_{11}=L_{G}=L_{R}=L_{22}=0.8529 \mu \mathrm{H} / \mathrm{m}$

$L_{m}=0.3725 \mu \mathrm{H} / \mathrm{m}$;

$L_{12}=L_{21}=L_{m}$

$C_{11}=C_{G}+C_{m}=C_{R}+C_{m}=C_{22}=46: 762 \mathrm{pF} / \mathrm{m} ;$

$C_{12}=C_{21}=-C_{m}=-18.036 \mathrm{pF} / \mathrm{m}$;

$L_{12} C_{11}+L_{22} C_{12}=L_{m}\left(C_{G}+C_{m}\right)-L_{R} C_{m}=0.3725 \times$ $\times 46.762-0.8529 \times 18.036=2.036 \neq 0$;

$L_{12} C_{22}+L_{11} C_{12}=L_{m}\left(C_{R}+C_{m}\right)-L_{G} C_{m}=0.3725 \times$ $\times 46.762-0.8529 \times 18.036=2.036 \neq 0$;

$\Delta_{C}=C_{11} C_{22}-C^{2}{ }_{12}=\left(C_{G}+C_{m}\right)\left(C_{R}+C_{m}\right)-C^{2}{ }_{m}=$ $=46.762^{2}-(-18.036)^{2} \approx 1861.3874>0$;

$\Delta_{L}=L_{G} L_{R}-L_{m}^{2}=L_{11} L_{22}-L^{2}{ }_{12}=$ $=0.8529^{2}-0.3725^{2} \approx 0.5887>0$;

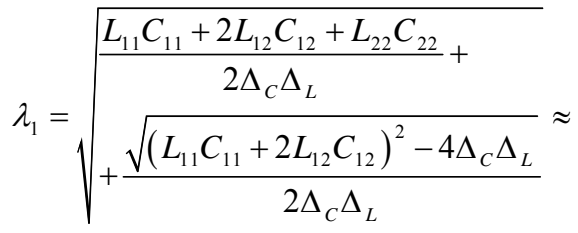

$\approx \sqrt{0.0321157} \approx 0.1792$ 


$$
\begin{aligned}
& \lambda_{2}=\sqrt{\frac{\frac{L_{11} C_{11}+2 L_{12} C_{12}+L_{22} C_{22}}{2 \Delta_{C} \Delta_{L}}-}{-\frac{\sqrt{\left(L_{11} C_{11}+2 L_{12} C_{12}\right)^{2}-4 \Delta_{C} \Delta_{L}}}{2 \Delta_{C} \Delta_{L}}}} \approx \\
& \approx \sqrt{0.0284} \approx 0.1686 \\
& \begin{array}{l}
L_{22}+\lambda_{1} \lambda_{2} \Delta_{L} C_{11}=0.8529+0.1792 \times 0.1686 \times \\
0.5887 \times
\end{array} \\
& \times 16.762 \approx 1.6846 \text {; } \\
& C_{22} \Delta_{L}+L_{11} \sqrt{\Delta_{L} \Delta_{C}}=46.762 \times 0.5887+ \\
& +0.8529 \sqrt{0.5887 \times 1861.3874} \approx 55.7546 \text {; } \\
& \Delta_{12}=\frac{\sqrt{D}\left(\sqrt{\Delta_{L} / \Delta_{C}}+R_{S} R_{F E}\right)+\lambda_{1}-\lambda_{2}}{L_{12} C_{11}+L_{22} C_{12}} \times \\
& \times\left(\left(C_{22} \Delta_{L}+L_{11} \sqrt{\Delta_{L} \Delta_{C}}\right) R_{N E}+\frac{L_{22}+\lambda_{1} \lambda_{2} \Delta_{L} C_{11}}{\lambda_{1} \lambda_{2}} R_{S}\right)= \\
& =0.427+24.051 R_{S} R_{N E}+3.5014 R_{N E}+19.6 R_{S} \text {; } \\
& \begin{aligned}
\Delta_{34} & =\frac{\sqrt{D}\left(\sqrt{\Delta_{L} / \Delta_{C}}+R_{\Lambda} R_{F E}\right)}{L_{12} C_{11}+L_{22} C_{12}}-\frac{\lambda_{1}-\lambda_{2}}{L_{12} C_{11}+L_{22} C_{12}} \times \\
& \times\left(\left(C_{22} \Delta_{L}+L_{11} \sqrt{\Delta_{L} \Delta_{C}}\right) R_{N E}+\frac{L_{22}+\lambda_{1} \lambda_{2} \Delta_{L} C_{11}}{\lambda_{1} \lambda_{2}} R_{\Lambda}\right)= \\
& =-\left(0.427+24.051 R_{\Lambda} R_{N E}+3.5014 R_{N E}+19.6 R_{\Lambda}\right) ;
\end{aligned} \\
& \gamma_{1}=\frac{1-\lambda_{1}^{2}\left(L_{11} C_{11}+L_{12} C_{12}\right)}{\lambda_{1}^{2}\left(L_{12} C_{11}+L_{22} C_{12}\right)}= \\
& =\frac{1-0.0321157(0.8529 \times 46.762-0.3725 \times 18.036)}{0.0321157(0.3725 \times 46.762-0.8529 \times 18.036)} \approx \\
& \approx \frac{1-1.0646}{0.065386} \approx-0.987979 \text {; } \\
& \gamma_{2}=\frac{1-\lambda_{2}^{2}\left(L_{11} C_{11}+L_{12} C_{12}\right)}{\lambda_{2}^{2}\left(L_{12} C_{11}+L_{22} C_{12}\right)}= \\
& =\frac{1-0.0284(0.8529 \times 46.762-0.3725 \times 18.036)}{0.0284(0.3725 \times 46.762-0.8529 \times 18.036)} \approx \\
& \approx \frac{1-0.94}{0.0578} \approx 1.038
\end{aligned}
$$

The inequalities from the main theorem are:

$$
\begin{aligned}
& \frac{\left|\lambda_{1} L_{12}+\left(L_{22} \lambda_{1}+R_{N E}\right) \gamma_{1}\right|}{\Delta_{12}} \breve{U}_{S}= \\
& \quad=\frac{\left|0.0667-0.98798\left(0.1528+R_{N E}\right)\right|}{0.427+\left(24.051 R_{S}+3.5014\right) R_{N E}+19.6 R_{S}} \breve{U}_{S} \leq \\
& \quad \leq \min \left\{I_{10}, I_{20}\right\}
\end{aligned}
$$

$$
\begin{aligned}
& \frac{\left|\lambda_{2} L_{12}+\left(L_{22} \lambda_{2}+R_{N E}\right) \gamma_{2}\right|}{\Delta_{12}} \breve{U}_{S}= \\
& \quad=\frac{\left|0.0628+1.038\left(0.1437+R_{N E}\right)\right|}{0.427+\left(24.051 R_{S}+3.5014\right) R_{N E}+19.6 R_{S}} \breve{U}_{S} \leq \\
& \quad \leq \min \left\{I_{10}, I_{20}\right\}
\end{aligned}
$$

In what follows we verify how the same data satisfy the conditions generated by the particular case under weak coupling assumptions. Indeed, the main system becomes

$$
\mid \begin{aligned}
& \left(C_{G}+C_{m}\right) \frac{\partial u_{G}(x, t)}{\partial t}+\frac{\partial i_{G}(x, t)}{\partial x}=0 \\
& -C_{m} \frac{\partial u_{G}(x, t)}{\partial t}+\left(C_{R}+C_{m}\right) \frac{\partial u_{R}(x, t)}{\partial t}+\frac{\partial i_{R}(x, t)}{\partial x}=0 \\
& L_{G} \frac{\partial i_{G}(x, t)}{\partial t}+\frac{\partial u_{G}(x, t)}{\partial x}=0 \\
& L_{m} \frac{\partial i_{G}(x, t)}{\partial t}+L_{R} \frac{\partial i_{R}(x, t)}{\partial t}+\frac{\partial u_{R}(x, t)}{\partial x}=0
\end{aligned}
$$

recalling that

$$
\begin{aligned}
& u_{1}(x, t)=u_{G}(x, t) ; \quad u_{2}(x, t)=u_{R}(x, t) \\
& i_{1}(x, t)=i_{G}(x, t) ; \quad i_{2}(x, t)=i_{R}(x, t) ; \\
& C_{11}=C_{G}+C_{m} ; \quad C_{12}=C_{21}=-C_{m} ; \quad C_{22}=C_{R}+C_{m} \\
& L_{11}=L_{G} ; \quad L_{12}=L_{21}=L_{m} ; \quad L_{22}=L_{R}
\end{aligned}
$$

or in a matrix form

$$
\left[\begin{array}{cccc}
C_{11} & 0 & 0 & 0 \\
C_{12} & C_{22} & 0 & 0 \\
0 & 0 & L_{11} & 0 \\
0 & 0 & L_{12} & L_{22}
\end{array}\right]+\left[\begin{array}{c}
\partial u_{1} / \partial t \\
\partial u_{2} / \partial t \\
\partial i_{1} / \partial t \\
\partial i_{2} / \partial t
\end{array}\right]+\left[\begin{array}{c}
\partial u_{1} / \partial x \\
\partial u_{2} / \partial x \\
\partial i_{1} / \partial x \\
\partial i_{2} / \partial x
\end{array}\right]=0
$$

Since

$$
\begin{aligned}
& \Delta_{C}=C_{11} C_{22}=\left(C_{G}+C_{m}\right)\left(C_{R}+C_{m}\right)>0 \\
& (L): \Delta_{L}=L_{11} L_{22}=L_{G} L_{R}>0
\end{aligned}
$$

it follows $|A|=\Delta_{C} \Delta_{L} \neq 0$

and therefore 


$$
A^{-1}=\left[\begin{array}{cccc}
\frac{C_{22}}{\Delta_{C}} & 0 & 0 & 0 \\
\frac{-C_{12}}{\Delta_{C}} & C_{11} \Delta_{C} & 0 & 0 \\
0 & 0 & \frac{L_{22}}{\Delta_{L}} & 0 \\
0 & 0 & \frac{-L_{12}}{\Delta_{L}} & L_{11} \Delta_{L}
\end{array}\right] .
$$

In view of

$$
\begin{aligned}
& B=\left[\begin{array}{cccc}
\frac{C_{22}}{\Delta_{C}} & 0 & 0 & 0 \\
\frac{-C_{12}}{\Delta_{C}} & C_{11} \Delta_{C} & 0 & 0 \\
0 & 0 & \frac{L_{22}}{\Delta_{L}} & 0 \\
0 & 0 & \frac{-L_{12}}{\Delta_{L}} & L_{11} \Delta_{L}
\end{array}\right] \times\left[\begin{array}{llll}
0 & 0 & 1 & 0 \\
0 & 0 & 0 & 1 \\
1 & 0 & 0 & 0 \\
0 & 1 & 0 & 0
\end{array}\right]= \\
& =\left[\begin{array}{cccc}
0 & 0 & \frac{C_{22}}{\Delta_{C}} & 0 \\
0 & 0 & \frac{-C_{12}}{\Delta_{C}} & C_{11} \Delta_{C} \\
\frac{L_{22}}{\Delta_{L}} & 0 & 0 & 0 \\
\frac{-L_{12}}{\Delta_{L}} & L_{11} \Delta_{L} & 0 & 0
\end{array}\right]
\end{aligned}
$$

we obtain

$$
\left[\begin{array}{c}
\frac{\partial u_{1}}{\partial t} \\
\frac{\partial u_{2}}{\partial t} \\
\frac{\partial i_{1}}{\partial t} \\
\frac{\partial i_{2}}{\partial t}
\end{array}\right]+\left[\begin{array}{cccc}
0 & 0 & \frac{C_{22}}{\Delta_{C}} & 0 \\
0 & 0 & \frac{-C_{12}}{\Delta_{C}} & C_{11} \Delta_{C} \\
\frac{L_{22}}{\Delta_{L}} & 0 & 0 & 0 \\
\frac{-L_{12}}{\Delta_{L}} & L_{11} \Delta_{L} & 0 & 0
\end{array}\right] \times\left[\begin{array}{c}
\frac{\partial u_{1}}{\partial t} \\
\frac{\partial u_{2}}{\partial t} \\
\frac{\partial i_{1}}{\partial t} \\
\frac{\partial i_{2}}{\partial t}
\end{array}\right]=0 .
$$

We find the eigenvectors

$$
H^{(k)}=\left(\xi_{1 k}, \xi_{2 k}, \xi_{3 k}, \xi_{4 k}\right)^{T} ;(k=1,2,3,4)
$$

of $\left(B^{-1}-\mu_{k} I\right) H^{(k)}=0 ; \mu_{k}=1 / \lambda_{k}$, where

$$
\left[\begin{array}{cccc}
0 & 0 & L_{11} & 0 \\
0 & 0 & L_{12} & L_{22} \\
C_{11} & 0 & 0 & 0 \\
C_{12} & C_{22} & 0 & 0
\end{array}\right]
$$

In view of $L_{12} C_{11}+L_{22} C_{12} \neq 0$ and $L_{12} C_{22}+L_{11} C_{12} \neq 0$ we obtain the roots of

$$
\left|B^{-1}-\mu I\right|=\left(L_{11} C_{11}-\mu^{2}\right)\left(L_{22} C_{22}-\mu^{2}\right)=0
$$

From the matrix

$$
\begin{aligned}
&\left(\begin{array}{cccc}
-\mu & 0 & L_{11} & 0 \\
0 & -\mu & L_{12} & L_{22} \\
C_{11} & 0 & -\mu & 0 \\
C_{12} & C_{22} & 0 & -\mu
\end{array}\right) \approx \\
& \approx\left(\begin{array}{cccc}
-\mu & 0 & L_{11} & 0 \\
0 & -\mu & L_{12} & L_{22} \\
0 & 0 & L_{11} C_{11}-\mu^{2} & 0 \\
0 & 0 & L_{11} C_{12}+L_{12} C_{22} & L_{22} C_{22}-\mu^{2}
\end{array}\right)
\end{aligned}
$$

we obtain for $\mu_{1}=-\mu_{3}=\sqrt{L_{11} C_{11}}$ the eigenvectors

$$
\begin{aligned}
& \xi_{12}=\frac{L_{12} C_{11}+L_{22} C_{12}}{L_{11} C_{12}+L_{12} C_{22}} \sqrt{\frac{L_{12}}{C_{11}}}, \quad \xi_{31}=\frac{L_{11} C_{11}-L_{22} C_{22}}{L_{11} C_{12}+L_{12} C_{22}} \\
& \xi_{41}=1, \quad \xi_{13}=-\frac{L_{11} C_{11}-L_{22} C_{22}}{L_{11} C_{12}+L_{12} C_{22}} \sqrt{\frac{L_{11}}{C_{11}}} \\
& \xi_{43}=1, \quad \xi_{23}=-\frac{L_{12} C_{11}+L_{22} C_{12}}{L_{11} C_{12}+L_{12} C_{22}} \sqrt{\frac{L_{11}}{C_{11}}} \\
& \xi_{33}=\frac{L_{11} C_{11}-L_{22} C_{22}}{L_{11} C_{12}+L_{12} C_{22}} .
\end{aligned}
$$

Similarly for $\mu_{2}=-\mu_{4}=\sqrt{L_{22} C_{22}} \Rightarrow$

$$
\left(\begin{array}{cccc}
-\sqrt{L_{22} C_{22}} & 0 & L_{11} & 0 \\
0 & -\sqrt{L_{22} C_{22}} & L_{12} & L_{22} \\
0 & 0 & L_{11} C_{11}-L_{22} C_{22} & 0 \\
0 & 0 & L_{11} C_{12}-L_{12} C_{22} & 0
\end{array}\right)
$$

we have

$$
\begin{aligned}
& \xi_{12}=0, \quad \xi_{22}=\sqrt{L_{22} / C_{22}}, \quad \xi_{32}=0, \quad \xi_{42}=1, \\
& \xi_{14}=0, \quad \xi_{24}=-\sqrt{L_{22} / C_{22}}, \quad \xi_{34}=0, \quad \xi_{44}=1 \text {. }
\end{aligned}
$$

Consequently, the transformation matrix is :

$$
\left(\begin{array}{cccc}
\frac{L_{11} C_{11}-L_{22} C_{22}}{L_{11} C_{12}+L_{12} C_{22}} \sqrt{\frac{L_{11}}{C_{11}}} & 0 & -\frac{L_{11} C_{11}-L_{22} C_{22}}{L_{11} C_{12}+L_{12} C_{22}} \sqrt{\frac{L_{11}}{C_{11}}} & 0 \\
\frac{L_{12} C_{11}+L_{22} C_{12}}{L_{11} C_{12}+L_{12} C_{22}} \sqrt{\frac{L_{12}}{C_{11}}} & \sqrt{\frac{L_{22}}{C_{22}}} & -\frac{L_{12} C_{11}+L_{22} C_{12}}{L_{11} C_{12}+L_{12} C_{22}} \sqrt{\frac{L_{12}}{C_{11}}} & -\sqrt{\frac{L_{22}}{C_{22}}} \\
\frac{L_{11} C_{11}-L_{22} C_{22}}{L_{11} C_{12}+L_{12} C_{22}} & 0 & \frac{L_{11} C_{11}-L_{22} C_{22}}{L_{11} C_{12}+L_{12} C_{22}} & 0 \\
1 & 1 & 1 & 1
\end{array}\right)
$$


And then in view of (12) we have

$$
\begin{aligned}
& u_{1}(x, t)=\frac{L_{11} C_{11}-L_{22} C_{22}}{L_{11} C_{12}+L_{12} C_{22}} \sqrt{\frac{L_{11}}{C_{11}}} I_{1}(x, t)- \\
& -\frac{L_{11} C_{11}-L_{22} C_{22}}{L_{11} C_{12}+L_{12} C_{22}} \sqrt{\frac{L_{11}}{C_{11}}} I_{3}(x, t) \text {; } \\
& u_{2}(x, t)=\frac{L_{12} C_{11}+L_{22} C_{12}}{L_{11} C_{12}+L_{12} C_{22}} \sqrt{\frac{L_{12}}{C_{11}}} I_{1}(x, t)+\sqrt{\frac{L_{22}}{C_{22}}} I_{2}(x, t)- \\
& -\frac{L_{12} C_{11}+L_{22} C_{12}}{L_{11} C_{12}+L_{12} C_{22}} \sqrt{\frac{L_{12}}{C_{11}}} I_{3}(x, t)-\sqrt{\frac{L_{22}}{C_{22}}} I_{4}(x, t) \text {; } \\
& i_{1}(x, t)=\frac{L_{11} C_{11}-L_{22} C_{22}}{L_{11} C_{12}+L_{12} C_{22}} I_{1}(x, t)+\frac{L_{11} C_{11}-L_{22} C_{22}}{L_{11} C_{12}+L_{12} C_{22}} I_{3}(x, t) \text {; } \\
& i_{2}(x, t)=I_{1}(x, t)+I_{2}(x, t)+I_{3}(x, t)+I_{4}(x, t) .
\end{aligned}
$$

If we take the specific parameters again from [9]

$$
\begin{aligned}
& L_{11}=L_{G}=L_{R}=L_{22}=0.8529 \mu \mathrm{H} / \mathrm{m} ; \\
& C_{11}=C_{G}+C_{m}=C_{R}+C_{m}=C_{22}=46.762 \mathrm{pF} / \mathrm{m}
\end{aligned}
$$

it is obvious that $L_{11} C_{11}-L_{22} C_{22}=0$. This implies $u_{1}(x, t)=u_{G}(x, t) \equiv 0$. The contradiction obtained shows the advantages of our method.

\section{Conclusion}

In this paper we presented the full derivation of the equations describing a 3-conductor transmission line terminated by linear loads. In such way, we extended the general method from [12] by shrinking the mixed problem for the hyperbolic system expressing TEM propagation lengthwise the lines to a functional system on the boundary. In result, by applying the fixed point method we can obtain in an explicit form the solution to the system of functional equations by successive approximations beginning with simple initial approximation. Our method is applicable to nonlinear boundary conditions too. Besides, in this paper we prove existenceuniqueness of a more general periodic solution and demonstrated the benefits of our method on the samples related to examinations of cross-talks. It should be noted that the method elaborated here can be applied to nonlinear boundary conditions.

\section{Acknowledgment}

This work is part of the research of L10S7 SynChaLab supported by Project "National Center of Mechatronics and Clean Technologies", Contract No. BG05M2OP001-1.001-0008/28.02.2018, funded by the Bulgarian Operational Program "Science and Education for Smart Growth 20142020".

\section{References:}

[1] C.A. Mead and L.A. Conway, Introduction to VLSI Systems. Addison-Wesley Publishing Co., Boston, MA, USA (1979).

[2] H.B. Bakoglu, Circuits, Interconnections, and Packaging for VLSI. Addison-Wesley Publishing Co. (1990).

[3] K.C. Gupta, Microstrip Lines and Slotlines. Artech House Publishers. 2nd ed. Norwood, MA, USA (1996).

[4] A.K. Goel, High-Speed VLSI Interconnections. John Wiley $\backslash \&$ Sons, Inc. 2nd ed. Hoboken, NJ, USA (2007).

[5] A.M. Dewey and S.W. Director, Principles of VLSI System Planning. A Framework for Conceptual Design. Springer US, Kluwer Academic Publishers. Boston/Dordrecht/London (1990).

[6] S. Rosenstark. Transmission Lines in Computer Engineering. Mcgraw-Hill. New York, NY, USA (1994).

[7] C.R. Paul. Introduction to Electromagnetic Compatibility. John Wiley \& Sons, Inc. 2nd ed. Hoboken, NJ, USA (2006).

[8] C.R. Paul. Analysis of Multi Conductor Transmission Lines. John Wiley \& Sons, Inc. 2nd ed. Hoboken, NJ, USA (2008).

[9] C.R. Paul. Solution of the Transmission-Line Equations under the Weak-Coupling Assumption. IEEE Transactions on Electrom. Compatibility. Vol 44. Iss. 3. pp. 413-423. (2002). 
[10] D.B. Jarvis. The Effects of Interconnections on High-Speed Logic Circuits. IEEE Transactions on Electrom. Compatibility. Vol EC-12. Iss. 5. pp. 476-487. (2006).

[11] V.G. Angelov. Calculation of Cross-Talks in Three-Conductor Transmission Line. Jubelee Scientific Session 2003, Higher School for Civil Engineering, “L. Karavelov”. pp. 93-99. (2003).

[12] V.G. Angelov. A Method for Analysis of Transmission Lines Terminated by Nonlinear Loads. Nova Science. New York (2014).

[13] V.G. Angelov. Fixed Points in Uniform Spaces and Applications. Cluj University Press. ClujNapoca, Romania (2009).

[14] D. Angelova, L. Georgiev, V. G. Angelov. A Goursat Problem for Sine-Gordon Equation, Proc. of 2008 Jubilee Scientific International Conference VSU. Vol. 1, pp. I-59-65. Sofia, Bulgaria (2008).

[15] D. Angelova, L. Georgiev. Lossless Transmission Lines Terminated by a Triode Generator Circuit, Thermal Energy and Power Engineering, Vol. 3, Iss. 4, pp. 284-309 (2014). 